\title{
Soft lubrication: the elastohydrodynamics of non-conforming and conforming contacts
}

\author{
J.M. Skotheim ${ }^{1,2}$ \& L. Mahadevan ${ }^{1,2}$ \\ ${ }^{1}$ Department of Applied Mathematics and Theoretical Physics, \\ Centre for Mathematical Sciences, Cambridge CB3 OWA, UK. \\ ${ }^{2}$ Division of Engineering and Applied Sciences, Harvard University, \\ Pierce Hall, 29 Oxford Street, Cambridge, MA 02138, USA
}

(Dated: October 5, 2018)

\begin{abstract}
We study the lubrication of fluid-immersed soft interfaces and show that elastic deformation couples tangential and normal forces and thus generates lift. We consider materials that deform easily, due to either geometry (e.g a shell) or constitutive properties (e.g. a gel or a rubber), so that the effects of pressure and temperature on the fluid properties may be neglected. Four different system geometries are considered: a rigid cylinder moving parallel to a soft layer coating a rigid substrate; a soft cylinder moving parallel to a rigid substrate; a cylindrical shell moving parallel to a rigid substrate; and finally a cylindrical conforming journal bearing coated with a thin soft layer. In addition, for the particular case of a soft layer coating a rigid substrate we consider both elastic and poroelastic material responses. For all these cases we find the same generic behavior: there is an optimal combination of geometric and material parameters that maximizes the dimensionless normal force as a function of the softness parameter $\eta=\frac{\text { hydrodynamic pressure }}{\text { elastic stiffness }}=\frac{\text { surface deflection }}{\text { gap thickness }}$ which characterizes the fluid-induced deformation of the interface. The corresponding cases for a spherical slider are treated using scaling concepts.
\end{abstract}




\section{INTRODUCTION}

The reduction of friction in practical applications has been studied since antiquity. Pictographs found in Uruk, located in modern day Iraq, have been dated to ca. 3000 B.C. and illustrate the transition from sleds to wheels (see Hamrock \& Dowson (1981) for a historical overview). While this advance certainly reduced friction, further reductions were possible upon the introduction of a viscous lubricating fluid in the axle joints. The theoretical underpinings of fluid lubrication in such geometries can be traced back to the work of Reynolds (1886), who studied the mechanics of fluid flow through a thin gap using an approximation to the Stokes' equations, now known as lubrication theory. Recent efforts in this technologically important problem have focused on modifications of Reynolds' lubrication theory to account for elastohydrodynamic effects (elastic surface deformation due to fluid pressure), piezoviscous behavior (lubricant viscosity change due to high pressure), and thermoviscous behavior (lubricant viscosity change due to frictional heating) (Dowson \& Higginson 1959; O’Donoghue, Brighton \&Hooke 1967; Conway \& Lee 1975; Hamrock \& Dowson 1981).

Inspired by a host of applications in physical chemistry, polymer physics and biolubrication, in this paper we focus on the elastohydrodynamics of soft interfaces, which deform easily thereby precluding piezoviscous and thermoviscous effects. There have been a number of works in these areas in the context of specific problems such as cartilage biomechanics (Grodzinsky, Lipshitz \& Glimcher 1978; Mow, Holmes \& Lai 1984; Mow \& Guo 2002), the motion of red blood cells in capillaries (Lighthill 1968; Fitz-Gerald 1969; Tözeren \& Skalak 1978; Secomb et al. 1986; Damiano et al. 1996; Secomb, Hsu \& Pries 1998; Feng \& Weinbaum 2000; Weinbaum et al. 2003), the elastohydrodynamics of rubber-like elastomers (Tanner 1966; Martin et al. 2002), polymer brushes (Klein, Perahia \& Warburg 1991; Sekimoto \& Leibler 1993) and vesicles (Abkarian, Lartigue \& Viallat 2002; Beaucourt, Biben \& Misbah 2004). Another related phenomenon is that of a bubble rising slowly near a wall; the bubble's surface deformation then leads to a lift force (Leal 1980; Takemura et al. 2002). Instead of focusing on specific applications, here we address a slightly different set of

questions: How can one generate lift between soft sliding surfaces to increase separation and reduce wear? What is the role of geometry in determining the behavior of such systems? How do material properties influence the elastohydrodynamics? Are there optimal combinations of the geometry and material properties that maximize the lift force? And finally, 
can the study of soft lubrication lead to improved engineering designs and be of relevance to real biological systems?

To address some of these questions we start with the simple case of two fluid-lubricated rigid non-conforming surfaces sliding past one another at a velocity $V$ as shown in figure 1. The viscous stresses and pressure gradient due to flow in the narrow contact zone are dominant. For a Newtonian fluid, the Stokes equations (valid in the gap) are reversible in time, $t$, so that the transformation $t \rightarrow-t$ implies the transformations of the velocity $V \rightarrow-V$ and the normal force $L \rightarrow-L$. In the vicinity of the contact region non-conforming surfaces are symmetric which implies that these flows are identical and therefore $L=0$. Elastohydrodynamics alters this picture qualitatively. In front of the slider the pressure is positive and pushes down the substrate, while behind the slider the pressure is negative and pulls up the substrate. As the solid deforms, the symmetry of the gap profile is broken leading to a normal force which pushes the cylinder away from the substrate.

This picture applies naturally to soft interfaces which arise either due to the properties of the material involved, as in the case of gels, or the underlying geometry, as in the case of thin shells. In §II we study the normal-tangential coupling of a non-conforming contact coated with a thin compressible elastic layer. If the gap profile prior to elastic deformation is parabolic in the vicinity of the contact, the contact is non-conforming. However, if a parabolic description prior to deformation is insufficient we refer to the contact as conforming; e.g. the degenerate case considered in $\$[\nabla]$ treats normal-tangential coupling of non-conforming contacts coated with a thick compressible elastic layer. In \$VI we consider the normal-tangential coupling of non-conforming contacts coated with an incompressible elastic layer. In $\$ \nabla I I$ we treat the normal-tangential coupling of non-conforming contacts coated with a thin compressible poroelastic layer which describes a biphasic material composed of an elastic solid matrix and a viscous fluid (Biot 1941). In \$VIII we study the normal-tangential coupling of a non-conforming contact where one solid is rigid and the other is a deformable cylindrical shell. In $₫ \mathbb{I X}$ we study a conforming contact: a journal bearing coated with a thin compressible elastic layer; Finally, in $₫[\mathbf{X}$ we treat the elastohydrodynamics of 3-dimensional flows using scaling analysis. Figure 2 provides an overview of the different geometries and elastic materials considered.

Our detailed study of a variety of seemingly distinct physical systems allows us clearly observe their similarities and to outline a robust set of features we expect to see in any 
soft contact. In all the cases studied the normal force $=$ contact area $\cdot$ characteristic hydrodynamic pressure $\cdot L(\eta)$, where $L(\eta)$ is the dimensionless lift and the softness parameter

$\eta=\frac{\text { hydrodynamic pressure }}{\text { elastic stiffness }} f$ (geometry) $=\frac{\text { elastic surface displacement }}{\text { characteristic gap thickness }}$. Tables 【 and 【] summarize our results for $\eta \ll 1$. Increasing $\eta$ increases the asymmetry of the gap profile which results in a repulsive elastohydrodynamic force, i.e. in the generation of lift forces. However, increasing $\eta$ also decreases the magnitude of the pressure distribution. The competition between symmetry breaking, which dominates for small $\eta$, and decreasing pressure, dominant at large $\eta$, produces an optimal combination of geometric and material parameters, $\eta_{\max }$, that maximize the dimensionless lift, $L$. Whether or not the normal force has a maximum depends on the control parameter: the normal force increases monotonically with the velocity, but has a maximum as a function of the effective elastic modulus of the system. This suggests that a judicious choice of material may aid in the generation of repulsive elastohydrodynamic forces thereby reducing friction and wear.

\section{FLUID LUBRICATION THEORY}

We consider a cylinder of radius $R$ moving at a velocity $V$ and rotating with angular frequency $\omega$ and immersed completely in a fluid of viscosity $\mu$ as shown in figure 11. The surfaces are separated by a distance $h(x)$, the gap profile, where the $x$-direction is parallel to the solid surface and the $z$-direction is perpendicular to it. We assume that the velocity and pressure field are two-dimensional and in the region of contact we use a parabolic approximation, valid for all non-conforming contacts, for the shape of the cylindrical surface in the absence of any elastic deformation. Then the total gap between the cylinder and the solid is given by

$$
h(x)=h_{0}\left(1+\frac{x^{2}}{2 h_{0} R}\right)+H(x),
$$

with $H$ begin the additional elastic deformation and $h_{0}$ the characteristic gap thickness in the absence of solid deformation. The size of the contact zone $\sqrt{2 h_{0} R}$, characterizes the horizontal size over which the lubrication forces are important. consistent with the parabolic approximation in (11). If $h_{0} \ll R$, the gap Reynolds number $\operatorname{Reg}=\frac{\rho V^{2} / l}{\mu V / h_{0}^{2}} \sim \frac{\rho V h_{0}^{3 / 2}}{\mu R^{1 / 2}} \ll \frac{\rho V R}{\mu}=$ Re, the nominal Reynolds number. Then, if Reg $\ll 1$ we can neglect the inertial terms and use the lubrication approximation (Reynolds 1886) to describe the hydrodynamics. For a 2-dimensional velocity field $\mathbf{v}=\left(v_{x}(x, z), v_{z}(x, z)\right)$ and a pressure field $p(x, z)$ the fluid stress 
tensor is

$$
\boldsymbol{\sigma}_{f}=\mu\left(\nabla \mathbf{v}+\nabla \mathbf{v}^{T}\right)-p \mathbf{I}
$$

Stress balance in the fluid, $\nabla \cdot \boldsymbol{\sigma}_{\boldsymbol{f}}=0$, yields

$$
\begin{array}{r}
0=\partial_{z} p \\
0=-\partial_{x} p+\mu \partial_{z z} v_{x}
\end{array}
$$

Mass conservation implies

$$
0=\partial_{x} v_{x}+\partial_{z} v_{z}
$$

The associated boundary conditions are

$$
\begin{aligned}
\left.v_{x}\right|_{z=-H}=-V,\left.\quad v_{x}\right|_{z=h_{0}+\frac{x^{2}}{2 R}} & =-\omega R, \\
\left.v_{z}\right|_{z=-H}=V \partial_{x} H,\left.\quad v_{z}\right|_{z=h_{0}+\frac{x^{2}}{2 R}} & =-x \omega, \\
\left.p\right|_{x \rightarrow \infty}=0,\left.\quad p\right|_{x \rightarrow-\infty} & =0,
\end{aligned}
$$

where we have chosen to work in a reference frame translating with the cylinder. We make the variables dimensionless with the following definitions

$$
\begin{aligned}
& x=\sqrt{2 h_{0} R} x^{\prime}, \quad z=h_{0} z^{\prime}, \quad p=p_{0} p^{\prime}=\frac{\sqrt{2 R} \mu(V-\omega R)}{h_{0}^{3 / 2}} p^{\prime}, \\
& \boldsymbol{\sigma}_{f}=p_{0} \boldsymbol{\sigma}_{f}^{\prime}=\frac{\sqrt{2 R} \mu(V-\omega R)}{h_{0}^{3 / 2}} \boldsymbol{\sigma}_{f}^{\prime}, \quad h=h_{0} h^{\prime}, \quad H=H_{0} H^{\prime}, \\
& v_{z}=(V-\omega R) \sqrt{\frac{h_{0}}{2 R}} v_{z}^{\prime}, \quad v_{x}=(V-\omega R) v_{x}^{\prime} .
\end{aligned}
$$

Here, $H_{0}\left(\frac{p_{0}}{E^{\prime}}, \frac{l_{2}}{l_{1}}, \frac{l_{3}}{l_{1}}, \ldots\right)$ is the characteristic scale of the deflection, where $E^{\prime}$ is the effective elastic modulus of the medium and $l_{i}$ are the length scales of the system. The pressure scaling follows from (3) and the fact that $x \sim \sqrt{h_{0} R}$. Then, after dropping the primes the dimensionless versions of equations (3)-(15) are

$$
\begin{array}{r}
0=\partial_{z} p, \\
0=-\partial_{x} p+\partial_{z z} v_{x}, \\
0=\partial_{x} v_{x}+\partial_{z} v_{z}, \\
\left.v_{x}\right|_{z=-\eta H}=\frac{-1}{1-\omega^{\prime}},\left.\quad v_{x}\right|_{z=1+x^{2}}=\frac{-\omega^{\prime}}{1-\omega^{\prime}}, \\
\left.v_{z}\right|_{z=-\eta H}=\frac{\eta \partial_{x} H}{1-\omega^{\prime}},\left.\quad v_{z}\right|_{z=1+x^{2}}=\frac{-2 x \omega^{\prime}}{1-\omega^{\prime}} . \\
\left.p\right|_{x \rightarrow \infty}=0,\left.\quad p\right|_{x \rightarrow-\infty}=0
\end{array}
$$


Here $\omega^{\prime}=\omega R / V$ characterizes the ratio of rolling to sliding, the softness parameter $\eta=$ $H_{0} / h_{0}$ characterizes the scale of the elastic deformation relative to the gap thickness, which is related to the compliance of the elastic material. The dimensionless version of the fluid stress tensor (2) is

$$
\boldsymbol{\sigma}_{f}=\left(\begin{array}{cc}
-p+2 \varepsilon^{2} \partial_{x} v_{x} & \varepsilon \partial_{z} v_{x}+\varepsilon^{3} \partial_{x} v_{z} \\
\varepsilon \partial_{z} v_{x}+\varepsilon^{3} \partial_{x} v_{z} & -p+2 \varepsilon^{2} \partial_{z} v_{z}
\end{array}\right) .
$$

where $\varepsilon=\sqrt{\frac{h_{0}}{2 R}}$. Solving (17) gives the Reynolds equation (Batchelor 1967)

$$
0=\partial_{x}\left(6 h+h^{3} \partial_{x} p\right)
$$

subject to

$$
p(\infty)=p(-\infty)=0 .
$$

Note that $\omega^{\prime}$ is scaled away. Here, $h$ is the gap profile given by (11) in dimensionless terms

$$
h(x)=1+x^{2}+\eta H(x) .
$$

To close the system we need to determine $\eta H(x)$, the elastic response to the hydrodynamic forces. This depends on the detailed geometry and constitutive behavior of the cylindrical contact. In the following sections we explore various configurations that allow us to explicitly calculate $\eta H$, thus allowing us to calculate the normal force on the cylinder

$$
L=\int_{\text {contact area }} p d A,
$$

and determine the elastohydrodynamic tangential-normal coupling. We note that when $\eta=0$, the contact is symmetric (11) so that the form of (9) implies that $p(-x)=-p(x)$ and $L=0$.

\section{ELASTIC 'LUBRICATION' THEORY: DEFORMATION OF A THIN ELAS- TIC LAYER}

In our first case, we consider a thin elastic layer of thickness $H_{l} \ll l_{c}$ coating the cylinder or the rigid wall or both, all of which are mathematically equivalent (figure 3). We first turn our attention to determining the surface deflection of the layer for an arbitrary applied traction. Throughout the analysis we assume that the surface deflection $H_{0} \ll H_{l}$ so that a 
linear elastic theory suffices to describe the material response. The stress tensor, $\boldsymbol{\sigma}_{s}$, for a linearly elastic isotropic material with Lamé coefficients $G$ and $\lambda$ is

$$
\boldsymbol{\sigma}_{s}=G\left(\nabla \mathbf{u}+\nabla \mathbf{u}^{T}\right)+\lambda \nabla \cdot \mathbf{u} \mathbf{I}
$$

where $\mathbf{u}=\left(u_{x}, u_{z}\right)$ is the displacement field, and $\mathbf{I}$ is the identity tensor. Stress balance in the solid implies

$$
\nabla \cdot \boldsymbol{\sigma}_{s}=0
$$

We make the equations dimensionless using

$$
z=H_{l} z^{\prime}, \quad x=\sqrt{2 h_{0} R} x^{\prime}, \quad u_{x}=h_{0} u_{x}^{\prime}, \quad u_{z}=h_{0} u_{z}^{\prime} \quad \boldsymbol{\sigma}_{s}=p_{0} \boldsymbol{\sigma}_{s}^{\prime} .
$$

We note that the length scale in the $z$-direction is the depth of the layer $H_{l}$; the length scale in the $x$-direction is the length scale of the hydrodynamic contact zone, $l_{c}=\sqrt{2 h_{0} R}$; the displacements $u_{x}$ and $u_{z}$ have been scaled with the characteristic gap thickness $h_{0}$; and the stress has been scaled using the hydrodynamic pressure scale following (66). We take the thickness of the solid layer to be small compared to the length scale of the contact zone with $\zeta=\frac{H_{l}}{\sqrt{2 h_{0} R}} \ll 1$, and restrict our attention to compressible elastic materials, where $G \sim \lambda$. Then, after dropping primes, the dimensionless 2-dimensional form of the stress tensor (13) is

$$
\boldsymbol{\sigma}_{s}=\frac{1}{\eta}\left(\begin{array}{cc}
\frac{\lambda}{2 G+\lambda} \partial_{z} u_{z}+\zeta \partial_{x} u_{x} & \frac{G}{2 G+\lambda} \partial_{z} u_{x}+\zeta \frac{G}{2 G+\lambda} \partial_{x} u_{z} \\
\frac{G}{2 G+\lambda} \partial_{z} u_{x}+\zeta \frac{G}{2 G+\lambda} \partial_{x} u_{z} & \partial_{z} u_{z}+\zeta \frac{\lambda}{2 G+\lambda} \partial_{x} u_{x}
\end{array}\right)
$$

Here

$$
\eta=\frac{p_{0}}{2 G+\lambda} \frac{H_{l}}{h_{0}}=\sqrt{2} \frac{\mu(V-\omega R)}{2 G+\lambda} \frac{H_{l} R^{1 / 2}}{h_{0}^{5 / 2}}
$$

is the softness parameter, a dimensionless number governing the relative size of the surface deflection to the undeformed gap thickness. Stress balance (14) yields

$$
\begin{gathered}
\partial_{z z} u_{x}+\zeta\left(1+\frac{\lambda}{G}\right) \partial_{x z} u_{z}+\zeta^{2}\left(2+\frac{\lambda}{G}\right) \partial_{x x} u_{x}=0 \\
\partial_{z z} u_{z}+\zeta \frac{G+\lambda}{2 G+\lambda} \partial_{x z} u_{x}+\zeta^{2} \frac{G}{2 G+\lambda} \partial_{x x} u_{z}=0
\end{gathered}
$$

so that to $O(\zeta)$ the leading order balance is

$$
\begin{gathered}
\partial_{z z} u_{x}=0 \\
\partial_{z z} u_{z}=0 .
\end{gathered}
$$


The normal unit vector to the soft interface is $\mathbf{n}=\left(-\left.\partial_{x} u_{z}\right|_{z=0}, 1\right)$, which in dimensionless form is

$$
\mathbf{n}=\left(-\left.\varepsilon \partial_{x} u_{z}\right|_{z=0}, 1\right)
$$

where $\varepsilon=\sqrt{\frac{h_{0}}{2 R}}$. The balance of normal traction on the solid-fluid interface yields

$$
\left.\boldsymbol{\sigma}_{f} \cdot \mathbf{n}\right|_{z=0}=\left.\boldsymbol{\sigma}_{s} \cdot \mathbf{n}\right|_{z=0},
$$

so that

$$
\left.\partial_{z} u_{x}\right|_{z=0}=0,\left.\quad \partial_{z} u_{z}\right|_{z=0}=-\eta p
$$

At the interface between the soft film and the rigid substrate, the no slip condition yields

$$
u_{z}(x,-1)=0, \quad u_{x}(x,-1)=0 .
$$

Solving (19), (22) and (23) gives us the displacement of the solid-fluid interface

$$
u_{x}(x, 0)=0, \quad u_{z}(x, 0)=-\eta p=-\eta H(x) .
$$

This linear relationship between the normal displacement and fluid pressure is known as the Winkler or 'mattress' elastic foundation model (Johnson 1985). In light of (24) we may write the gap profile (111) as

$$
h=1+x^{2}+\eta p
$$

Equations (9), (10) and (25) form a closed system for the elastohydrodynamic response of a thin elastic layer coating a rigid cylinder. We note that Lighthill (1968) found a similar set of equations while studying the flow of a red blood cell through a capillary. However, his model's axisymmetry proscribed the existence of a force normal to the flow.

When $\eta \ll 1$ we can employ a perturbation analysis to find the lift force experienced by the cylinder. We use an expansion of the form

$$
p=p^{(0)}+\eta p^{(1)}+O\left(\eta^{2}\right), \quad h=h^{(0)}+\eta h^{(1)}+O\left(\eta^{2}\right),
$$

to find

$$
\begin{aligned}
\eta^{0}: \partial_{x}\left\{6 h^{(0)}+\left[h^{(0)}\right]^{3} \partial_{x} p^{(0)}\right\} & =0, \\
\eta^{1}: \partial_{x}\left\{6 h^{(1)}+3\left[h^{(0)}\right]^{2} h^{(1)} \partial_{x} p^{(0)}+\left[h^{(0)}\right]^{3} \partial_{x} p^{(1)}\right\} & =0,
\end{aligned}
$$


where

$$
h^{(0)}=1+x^{2}, \quad h^{(1)}=p^{(0)},
$$

subject to the boundary conditions

$$
p^{(0)}(\infty)=p^{(0)}(-\infty)=p^{(1)}(\infty)=p^{(1)}(-\infty)=0 .
$$

Solving (27)-(30) yields

$$
p=\frac{2 x}{\left(1+x^{2}\right)^{2}}+\eta \frac{3\left(3-5 x^{2}\right)}{5\left(1+x^{2}\right)^{5}}+O\left(\eta^{2}\right),
$$

so that

$$
L=\int p d x=\frac{3 \pi}{8} \eta .
$$

In dimensional form the lift force per unit length is

$$
L=\frac{3 \sqrt{2} \pi}{8} \frac{p_{0}^{2}}{2 G+\lambda} \frac{H_{l} \sqrt{R}}{\sqrt{h_{0}}}=\frac{3 \sqrt{2} \pi}{4} \frac{\mu^{2}(V-\omega R)^{2}}{2 G+\lambda} \frac{H_{l} R^{3 / 2}}{h_{0}^{7 / 2}},
$$

as reported in Skotheim \& Mahadevan (2004b). The same scaling was found by Sekimoto \& Leibler (1993), but with a different prefactor owing to a typographical error. When $\eta=O(1)$, the system (9), (10) and (25) is solved numerically using a continuation method (Doedel et al. 2004) with $\eta$ as the continuation parameter. In figure 4 we show the pressure distribution

$p(x)$, and gap $h(x)$ as a function of $\eta$. For $\eta \ll 1, p\left(-\frac{9 \eta}{10}\right)=0$. As $\eta$ increases, $h$ increases and the asymmetric gap profile begins to resemble that of a tilted slider bearing, which is well known to generate lift forces. However, an increase in the gap thickness also decreases the peak pressure $\sim \mu V R^{1 / 2} / h_{0}^{3 / 2}$ (see figure 4 h). The competition between symmetry breaking,

dominant for $\eta \lesssim 1$, and decreasing pressure, dominant for $\eta \gtrsim 1$, produces a maximum scaled lift force at $\eta=2.06$. In dimensional terms, this implies that the lift as a function of the effective modulus $2 G+\lambda$ will have a maximum, however, the lift as a function of the relative motion between the two surfaces $V-\omega R$ increases monotonically (see figure 5b). In fact, (33) shows that the dimensional lift increases as $(V-\omega R)^{2}$ for $\eta \ll 1$.

\section{DEGENERATE CONTACT}

In this section we consider the case where the parabolic approximation in the vicinity of the contact breaks down. Since rotation changes the nature of the contact region for such 
interfaces, we consider only a purely sliding motion with $\omega=0$. We assume that the gap thickness is described by

$$
h=h_{0}\left(1+\frac{x^{2 n}}{h_{0} R^{2 n-1}}\right)+H(x),
$$

where $n=2,3, \ldots$ characterizes the geometric nature of the contact and the contact length is $l_{c} \sim\left(h_{0} R^{2 n-1}\right)^{1 / 2 n}$. We note that we always focus on symmetric contacts. We make the variables dimensionless using the following scalings

$$
\begin{array}{r}
h=h_{0} h^{\prime}, \quad x=l^{*} x^{\prime}=h_{0}^{\frac{1}{2 n}} R^{1-\frac{1}{2 n}} x^{\prime}, \\
p=p^{*} p^{\prime}=\mu V \frac{R^{1-\frac{1}{2 n}}}{h_{0}^{2-\frac{1}{2 n}}} p^{\prime} .
\end{array}
$$

In III we have seen that for a thin compressible soft layer the pressure and and surface deflection can be linearly related by (24) so that the scale of the deflection is $h_{0} \eta=\frac{p_{0} H_{l}}{2 G+\lambda}$. To find the scale of the deflection for the degenerate contact described by (34), we replace $p_{0}$ with the appropriate pressure scale $p^{*}$ so that

$$
H=\frac{p^{*}}{2 G+\lambda} H_{l} H^{\prime}=\frac{\mu V}{2 G+\lambda} \frac{H_{l} R^{1-\frac{1}{2 n}}}{h_{0}^{2-\frac{1}{2 n}}},
$$

and the size of the deformation relative to the gap size is governed by the dimensionless group

$$
\eta=\frac{p^{*}}{2 G+\lambda} \frac{H_{l}}{h_{0}}=\frac{\mu V}{2 G+\lambda} \frac{H_{l} R^{1-\frac{1}{2 n}}}{h_{0}^{3-\frac{1}{2 n}}} .
$$

Then the dimensionless version of the gap thickness profile (34) is

$$
h=1+x^{2 n}+\eta p
$$

As in $₫$ III we employ a perturbation expansion for the pressure field in the parameter $\eta$ $(\ll 1)$ to solve (91), (10) and (38) and find the pressure field and the lift for small $\eta$. This yields $p=p_{0}+\eta p_{1}$ and gives the following dimensionless result

$$
L_{n=2}=\frac{351 \pi}{784 \sqrt{2}} \eta, \quad L_{n=3}=0.8859 \eta
$$

where we have not shown the pressure distribution due to its unwieldy size. In dimensional terms, the normal force reads as

$$
\begin{gathered}
L_{n=2}=\frac{351 \pi}{784 \sqrt{2}} \frac{p^{* 2}}{2 G+\lambda} \frac{H_{l} l^{*}}{h_{0}}=\frac{351 \pi}{784 \sqrt{2}} \frac{\mu^{2} V^{2} H_{l} R^{9 / 4}}{(2 G+\lambda) h_{0}^{17 / 4}}, \\
L_{n=3}=0.8859 \frac{p^{* 2}}{2 G+\lambda} \frac{H_{l} l^{*}}{h_{0}}=0.8859 \frac{\mu^{2} V^{2} H_{l} R^{5 / 2}}{(2 G+\lambda) h_{0}^{9 / 2}} .
\end{gathered}
$$


The results for $n=2$ and $n=3$ are shown in figures [6 and 7. One noteworthy feature of a degenerate contact is that the torque experienced by the slider arises from the fluid pressure rather that the shear force because the normal to the surface no longer passes through the center of the object as it would for a cylinder or sphere. The ratio of the torque due to shear to the torque due to the pressure is

$$
\frac{\text { shear torque }}{\text { pressure torque }} \sim \frac{\mu V R / h_{0}}{p l_{c}} \sim\left(\frac{h_{0}}{R}\right)^{1-\frac{1}{n}} \ll 1 .
$$

Hence, the dominant contribution to the torque is due to the pressure and

$$
\Gamma=\int p x d A
$$

For $\eta \ll 1, p=p_{0}+O(\eta)$ so that

$$
\begin{array}{r}
n=2: \quad p_{0}=\frac{6 x}{7\left(1+x^{4}\right)^{2}}, \quad \Gamma=\frac{3 \pi}{14 \sqrt{2}}, \\
n=3: \quad p_{0}=\frac{6 x}{11\left(1+x^{6}\right)^{2}}, \quad \Gamma=\frac{\pi}{11}, \\
n=m: \quad p_{0}=\frac{6 x}{(4 m-1)\left(1+x^{2 m}\right)^{2}}, \quad \Gamma=\frac{3 \pi(2 m-3) \csc \frac{3 \pi}{2 m}}{m^{2}(4 m-1)} .
\end{array}
$$

\section{SOFT SLIDER}

To contrast our result for a thin layer with that for a soft slider, we consider the case where the entire cylindrical slider of length $l$ and radius $R$ is made of a soft material with Lamé coefficients $G$ and $\lambda$. Equivalently, we could have a rigid slider moving above a soft semiinfinite half space. Since the deformation is no longer locally determined by the pressure we use a Green's function approach to determine the response to the hydrodynamic pressure. Following Davis, Serayssol \& Hinch (1986), we use the Green's function for a point force on a half space since the scale of the contact zone, $l_{c} \sim \sqrt{h_{0} R} \ll R$, the cylinder radius. Following Landau \& Lifshitz (1970) we write the deformation at the surface due to a pressure field $p(x, y)$ as

$$
H(x, y)=\frac{\lambda+2 G}{4 \pi G(\lambda+G)} \int \frac{p\left(x^{\prime}, y^{\prime}\right) d x^{\prime} d y^{\prime}}{\sqrt{\left(x^{\prime}-x\right)^{2}+\left(y^{\prime}-y\right)^{2}}},
$$

with $x, y$ as defined in figure 8 . Neglecting end effects so the pressure is a function of $x$ only, we integrate (45) over $y^{\prime}$ to get

$$
\begin{aligned}
H(x) & =\frac{\lambda+2 G}{4 \pi G(\lambda+G)} \int_{-\infty}^{\infty}\left[p\left(x^{\prime}\right) \int_{-l}^{l} \frac{d y^{\prime}}{\sqrt{\left(x^{\prime}-x\right)^{2}+\left(y^{\prime}-y\right)^{2}}}\right] d x^{\prime} \\
& =\quad \frac{\lambda+2 G}{4 \pi G(\lambda+G)} \int_{-\infty}^{\infty} \log \left[\frac{4\left(l^{2}-y^{2}\right)}{\left(x-x^{\prime}\right)^{2}}\right] p\left(x^{\prime}\right) d x^{\prime}
\end{aligned}
$$


To make the equations dimensionless we employ the following scalings

$$
\begin{gathered}
x=\sqrt{2 h_{0} R} x^{\prime}, \quad y=l y^{\prime}, \quad h=h_{0} h^{\prime}, \\
H=h_{0} H^{\prime}, \quad p=p_{0} p^{\prime}=\frac{\sqrt{2 R} \mu(V-\omega R)}{h_{0}^{3 / 2}} p^{\prime},
\end{gathered}
$$

so that the dimensionless gap thickness (11) now reads

$$
h=1+x^{2}+\eta \int_{-\infty}^{\infty} d x^{\prime} p\left(x^{\prime}\right) \log \left[\frac{Y}{\left(x-x^{\prime}\right)^{2}}\right]
$$

where

$$
\eta=\frac{1}{2 \pi} \frac{\mu(V-\omega R)(\lambda+2 G)}{G(\lambda+G)} \frac{R}{h_{0}^{2}}, \quad Y=\frac{2 l^{2}}{R h_{0}}\left(1-y^{2}\right) .
$$

Comparing with our softness parameter for a thin section we see that

$$
\frac{\eta_{\text {thin layer }}}{\eta_{\text {Soft slider }}}=2 \pi \sqrt{2} \frac{G(G+\lambda)}{(2 G+\lambda)^{2}} \frac{H_{l}}{\sqrt{h_{0} R}} \sim \frac{H_{l}}{\sqrt{h_{0} R}} \ll 1 \text {, }
$$

i.e. a thin layer is stiffer than a half space made from the same material by the geometric factor $\frac{\sqrt{h_{0} R}}{H_{l}}$. For small $\eta$ we write $p=p_{0}+\eta p_{1}$ where $p_{0}=\frac{2 x}{\left(1+x^{2}\right)^{2}}$ as in (31). Substituting into (48) yields

$$
h=1+x^{2}+\eta \frac{2 \pi x}{1+x^{2}}+O\left(\eta^{2}\right) .
$$

To order $\eta$, (9) and (10) yield the equations for the perturbation pressure, $p_{1}$ :

$$
\begin{array}{r}
\eta: \quad 0=\partial_{x}\left[\left(1+x^{2}\right)^{3} \partial_{x} p_{1}+\frac{24 \pi x\left(x^{2}-1\right)}{\left(1+x^{2}\right)^{2}}\right] \\
p_{1}(-\infty)=p_{1}(\infty)=0
\end{array}
$$

which has the solution

$$
p_{1}=\frac{2 \pi\left(2 x^{2}-1\right)}{\left(1+x^{2}\right)^{4}}
$$

Hence the dimensionless lift force is

$$
L=\eta \int p_{1} d x=\eta \int \frac{2 \pi\left(2 x^{2}-1\right)}{\left(1+x^{2}\right)^{4}} d x=\frac{3 \pi^{2}}{8} \eta .
$$

In dimensional terms the lift force is

$$
L=\frac{3 \pi}{8} \frac{\mu^{2}(V-\omega R)^{2}(\lambda+2 G)}{G(\lambda+G)} \frac{R^{2}}{h_{0}^{3}} .
$$

Comparing this expression with that for the case of a thin elastic layer, equation (33), we see that confinement acts to reduce deformation and hence reduce the lift in the small 
deflection, $\eta \ll 1$, regime. When $\eta=O(1)$ we solve (9), (10) and (48) for $p(x)$ using an iterative procedure. First, we guess an initial gap profile $h_{\text {old }}$ and use a shooting algorithm to calculate the pressure distribution. The new pressure distribution is then used in equation (48) with $Y=1000$ (corresponding to a very long cylinder) to calculate a new gap profile,

$h_{\text {new }}$. If $\int_{-10}^{10}\left(h_{\text {old }}-h_{\text {new }}\right)^{2} d x<10^{-6}$ the calculation is stopped, else we set $h_{\text {old }}=h_{\text {new }}$ and iterate. The results are shown in figures 9 and 10, and not surprisingly they have the same qualitative features discussed previously, i.e. for $\eta \ll 1, L \sim \eta ; L$ shows a maximum at $\eta=0.25$ and decreases when $\eta>0.25$. The reasons for this are the same as before, i.e. the competing effects of an increase in the gap thickness and the increased asymmetry of the contact zone.

\section{INCOMPRESSIBLE LAYER}

In contrast to compressible layers, an incompressible layer (e.g. one made of an elastomer) can deform only via shear. For thin layers, incompressibility leads to a geometric stiffening that qualitatively changes the nature of the elastohydrodynamic problem (Johnson 1985). To address this problem in the most general case, we use a Green's function approach. The constitutive behavior for an incompressible linearly elastic solid is

$$
\boldsymbol{\sigma}=G\left(\nabla \mathbf{u}+\nabla \mathbf{u}^{T}\right)-p_{s} \mathbf{I}
$$

where $\mathbf{u}=\left(u_{x}, u_{y}, u_{z}\right)$ is the displacement and $p_{s}$ is the pressure in the solid. Mechanical equilibrium in the solid implies $\nabla \cdot \boldsymbol{\sigma}=0$, i.e.

$$
\begin{aligned}
& 0=-\partial_{x} p_{s}+G \nabla^{2} u_{x} \\
& 0=-\partial_{y} p_{s}+G \nabla^{2} u_{y} \\
& 0=-\partial_{z} p_{s}+G \nabla^{2} u_{z} .
\end{aligned}
$$

Incompressibility of the solid implies

$$
0=\nabla \cdot \mathbf{u}=\partial_{x} u_{x}+\partial_{y} u_{y}+\partial_{z} u_{z}
$$


For the Green's function associated with a point force, $\left.\sigma_{z z}\right|_{z=0}=-f \delta(x) \delta(y)$ where $\delta$ is a delta function, the boundary conditions are

$$
\begin{gathered}
u_{x}=u_{y}=u_{z}=0 \quad \text { at } \quad z=-H_{l}, \\
\sigma_{x z}=\sigma_{x y}=0 \quad \text { at } \quad z=0, \\
\sigma_{z z}=-f \delta(x) \delta(y) \quad \text { at } \quad z=0 .
\end{gathered}
$$

We solve the boundary value problem (57)-(59) by using a 2-D Fourier transforms defined as

$$
u_{x}=\int_{-\infty}^{\infty} \int_{-\infty}^{\infty} \hat{u}_{x}\left(k_{x}, k_{y}, z\right) e^{-i\left(k_{x} x+k_{y} y\right)} d k_{x} d k_{y}, \ldots
$$

Then equations (57)-(59) in Fourier space are

$$
\begin{array}{r}
0=i k_{x} \hat{p}_{s}+G\left(-k_{x}^{2} \hat{u}_{x}-k_{y}^{2} \hat{u}_{x}+\partial_{z z} \hat{u}_{x}\right), \\
0=i k_{y} \hat{p}_{s}+G\left(-k_{x}^{2} \hat{u}_{y}-k_{y}^{2} \hat{u}_{y}+\partial_{z z} \hat{u}_{y}\right), \\
0=-\partial_{z} \hat{p}_{s}+G\left(-k_{x}^{2} \hat{u}_{z}-k_{y}^{2} \hat{u}_{z}+\partial_{z z} \hat{u}_{z}\right), \\
0=-i k_{x} \hat{u}_{x}-i k_{y} \hat{u}_{y}+\partial_{z} \hat{u}_{z},
\end{array}
$$

subject to the boundary conditions

$$
\begin{array}{r}
\hat{\sigma}_{x z}=0=\partial_{z} \hat{u}_{x}-i k_{x} \hat{u}_{z} \text { at } z=0, \\
\hat{\sigma}_{y z}=0=\partial_{z} \hat{u}_{y}-i k_{y} \hat{u}_{z} \text { at } z=0, \\
\hat{\sigma}_{z z}=-f=-\hat{p}_{s}+2 G \partial_{z} \hat{u}_{z} \text { at } z=0, \\
\hat{u}_{x}=\hat{u}_{y}=\hat{u}_{z}=0 \text { at } z=-H_{l} .
\end{array}
$$

Solving (61) and (62) for $\hat{u}_{z}(z=0)$ we find

$$
\hat{u}_{z}\left(k_{x}, k_{y}, 0\right)=f \frac{2 H_{l}-\frac{\sinh \left(2 H_{l} \sqrt{k_{x}^{2}+k_{y}^{2}}\right)}{\sqrt{k_{x}^{2}+k_{y}^{2}}}}{2 G\left[1+2 H_{l}^{2}\left(k_{x}^{2}+k_{y}^{2}\right)+\cosh \left(2 H_{l} \sqrt{k_{x}^{2}+k_{y}^{2}}\right)\right.} .
$$

Since this corresponds to a radially symmetric integral kernel we can take $q=\sqrt{k_{x}^{2}+k_{y}^{2}}$ and use the Hankel transform, $u_{z}=\frac{1}{2 \pi} \int_{0}^{\infty} J_{0}(r q) q \hat{u}_{z} d q$, to find the surface displacement (Gladwell 1980):

$$
\left.u_{z}(r)\right|_{z=0}=\frac{f}{4 \pi G} \int_{0}^{\infty} J_{0}(r q) \frac{2 H_{l} q-\sinh \left(2 H_{l} q\right)}{1+2 H_{l}^{2} q^{2}+\cosh \left(2 H_{l} q\right)} d q=\frac{f}{4 \pi G H_{l}} W(r),
$$


where $r=\sqrt{x^{2}+y^{2}}$ and $J_{0}(r q)$ is a Bessel function of the first kind. To see the form of the surface displacement, we integrate (64) numerically and show the dimensionless function $W(r)$ in figure 11]. The surface displacement $H$ for a pressure distribution $p(x)$ is found using the Green's function for a point force (64), so that

$$
H=-\int_{-\infty}^{\infty} \frac{p\left(x^{*}\right)}{4 \pi G}\left\{\int_{-\infty}^{\infty}\left[\int_{0}^{\infty} J_{0}(r q) \frac{2 H_{l} q-\sinh 2 H_{l} q}{1+2 H_{l}^{2} q^{2}+\cosh 2 H_{l} q} d q\right] d y^{*}\right\} d x^{*}
$$

where $r=\sqrt{\left(x-x^{*}\right)^{2}+\left(y-y^{*}\right)^{2}}$. In terms of dimensionless variables with the following definitions

$$
\begin{array}{r}
p=p_{0} p^{\prime}, q=\frac{q^{\prime}}{\sqrt{2 h_{0} R}}, x=\sqrt{2 h_{0} R} x^{\prime}, x^{*}=\sqrt{2 h_{0} R} x^{\prime *}, \\
y=\sqrt{2 h_{0} R} y^{\prime}, y^{*}=\sqrt{2 h_{0} R} y^{\prime *}, r=\sqrt{2 h_{0} R} r^{\prime}, \quad h=h_{0} h^{\prime}, \\
H=\frac{p_{0} \sqrt{2 h_{0} R}}{4 \pi G} H^{\prime}=\frac{\mu(V-\omega R)}{2 \pi G} \frac{R}{h_{0}} H^{\prime},
\end{array}
$$

equation (65) may be rewritten (after dropping primes) as

$$
H=-\int_{-\infty}^{\infty} p\left(x^{*}\right)\left\{\int_{-\infty}^{\infty}\left[\int_{0}^{\infty} J_{0}(r q) \frac{2 \zeta q-\sinh 2 \zeta q}{1+2 \zeta^{2} q^{2}+\cosh 2 \zeta q} d q\right] d y^{*}\right\} d x^{*}
$$

where $r=\sqrt{\left(x-x^{*}\right)^{2}+\left(y-y^{*}\right)^{2}}$, and the dimensionless group $\zeta=\frac{H_{l}}{\sqrt{2 h_{0} R}}$ is the ratio of the layer thickness to the size of the contact zone. To understand the form of (67) we consider the response of a line force $p\left(x^{*}\right)=\delta\left(x^{*}\right)$ and show the results graphically in figure 11p. Moving on to the case at hand, that of a parabolic contact of a cylinder of length $2 l$, we write the dimensionless gap thickness as

$$
h=1+x^{2}+\eta H, \text { where } \eta=\frac{1}{2 \sqrt{2} \pi} \frac{p_{0}}{G} \sqrt{\frac{R}{h_{0}}}=\frac{1}{2 \pi} \frac{\mu(V-\omega R)}{G} \frac{R}{h_{0}^{2}}
$$

For $\eta \ll 1$ we can expand the pressure $p$ in powers of $\eta$ and carry out a perturbation analysis as in $₫$ III. This yields a linear relation between the dimensionless lift, $L$, and the scale of the deformation: $L=C_{i}(\zeta) \eta$, where $C_{i}(\zeta)$ is shown in figure 12 $\mathrm{d}$. The dimensional lift per unit length is

$$
L=\frac{C_{i}(\zeta)}{2 \pi} \frac{\mu^{2}(V-\omega R)^{2}}{G} \frac{R^{2}}{h_{0}^{3}} .
$$

As $\zeta \rightarrow 0$, we approach the limit of an infinitely thick layer, in which case there is no stiffening due to incompressibility, so that $C(\zeta) \rightarrow \frac{3 \pi^{2}}{8}$, which is the result for an infinitely thick layer. To study the effects of confinement on a thick layer, i.e. $\zeta \gg 1$, we approximate 
(67) as

$$
H \approx-\int_{-\infty}^{\infty} p\left(x^{*}\right) \int_{-l^{\prime}}^{l^{\prime}}\left\{\int_{0}^{\infty} J_{0}(r q)\left[1-\left(2+4 \zeta q+\zeta^{2} q^{2}\right) e^{-2 \zeta q}\right] d q\right\} d y^{*} d x^{*}
$$

where we are now integrating $y^{*}$ over the dimensionless length of the cylinder $l^{\prime}=\frac{l}{\sqrt{2 h_{0} R}} \gg 1$, since the interactions for deep layers are not limited by confinement effects. Evaluating (170) yields

$$
H \approx \int_{-\infty}^{\infty} p\left(x^{*}\right)\left\{\int_{-l^{\prime}}^{l^{\prime}}\left[-\frac{1}{r}-\frac{2 r^{4}+20 r^{2} \zeta^{2}+96 \zeta^{4}}{\left(r^{2}+4 \zeta^{2}\right)^{5 / 2}}\right] d y^{*}\right\} d x^{*} .
$$

Integrating (171) with respect to $y^{*}$ and keeping the leading order terms in $l^{\prime}$ yields

$$
\begin{array}{r}
H \approx \int_{-\infty}^{\infty} \frac{2 x^{*}}{\left(1+x^{* 2}\right)^{2}}\left\{\log \left[\frac{4\left(l^{\prime 2}-y^{2}\right)}{\left(x-x^{*}\right)^{2}}\right]+2 \log \left[\frac{\left(x-x^{*}\right)^{2}+4 \zeta^{2}}{4\left(l^{2}+y^{2}\right)}\right]\right. \\
\left.-\frac{8 \zeta^{2}\left[\left(x-x^{*}\right)^{2}+12 \zeta^{2}\right]}{\left[\left(x-x^{*}\right)^{2}+4 \zeta^{2}\right]^{2}}\right\} d x^{*}
\end{array}
$$

where we have used the leading order pressure (31) to evaluate $p\left(x^{*}\right)=\frac{2 x^{*}}{\left(1+x^{* 2}\right)^{2}}+\eta p^{(1)}$. We integrate (72) with respect to $x^{*}$ to find

$$
H \approx \frac{24 \pi x\left(x^{2}-1\right)}{\left(1+x^{2}\right)^{2}}-\frac{4 \pi x\left[x^{4}+2 x^{2}\left(1+6 \zeta+6 \zeta^{2}\right)+(1+2 \zeta)^{2}\left(1+8 \zeta+24 \zeta^{2}\right)\right]}{\left[x^{2}+(1+2 \zeta)^{2}\right]^{3}}
$$

As in $₫$ III] equations (28) and (30) yields the system of equations to be solved for the pressure perturbation $p^{(1)}$ :

$$
\begin{array}{r}
\partial_{x}\left[6 H+3\left(1+x^{2}\right)^{2} H+\left(1+x^{2}\right)^{3} \partial_{x} p^{(1)}\right]=0 \\
p^{(1)}(-\infty)=p^{(1)}(\infty)=0 .
\end{array}
$$

Solving (73)-(74) yields

$$
p^{(1)}=\frac{2 \pi\left(2 x^{2}-1\right)}{\left(1+x^{2}\right)^{4}}-\frac{\pi\left[12 \zeta^{2}-20 \zeta+30-x^{2}\left(45-60 \zeta+36 \zeta^{2}\right)+45 x^{4}\right]}{2 \zeta^{4}\left(1+x^{2}\right)^{3}},
$$

which we integrate with respect to $x$ to find the dimensionless lift

$$
L=\eta p^{(1)} d x=\eta C_{i}=\eta \frac{3 \pi^{2}}{8}\left(1-\frac{30}{\zeta^{4}}\right)+O\left(\zeta^{-5}\right)
$$

On the other hand, as $\zeta \rightarrow 0$ we approach the limit where the contact length is much larger than the layer thickness leading to geometric stiffening. Due to solid incompressibility the layer may only deform via shear and the dominant term of the strain is $\nabla \mathbf{u} \sim \frac{u_{x}}{H_{l}}$. Since $\nabla \cdot \mathbf{u}=0$ we see that $\frac{u_{x}}{l_{c}} \sim \frac{H}{H_{l}} \rightarrow \frac{u_{x}}{H_{l}} \sim \frac{l_{c} H}{H_{l}^{2}}$. Balancing the strain energy $\int G(\nabla \mathbf{u})^{2} d V \sim$ 
$G\left(\frac{l_{c} H}{H_{l}}\right) H_{l} l_{c}$ with the work done by the pressure $p_{0} H l_{c}$ yields $H \sim \frac{p_{0} H_{l}^{3}}{G l_{c}^{2}}$ so that $\eta_{\zeta \rightarrow \infty} \sim$ $\frac{p_{0} H_{l}^{3}}{G l_{c}^{2} h_{0}}$. For a thin incompressible layer the characteristic deflection is reduced by an amount $\frac{\eta_{\zeta \rightarrow \infty}}{\eta} \sim \zeta^{-3}$ so that $\lim _{\zeta \rightarrow \infty} C_{i}(\zeta) \sim \zeta^{3} . C_{i}$ is displayed in figure 12a. For intermediate values of $\zeta$, we computed the results numerically.

The nonlinear problem arising for $\eta=O(1)$ is solved using (91), (10), (67) and (68). We first guess an initial gap profile $h_{\text {old }}$, then a shooting algorithm is employed to calculate the pressure distribution. The new pressure distribution is then used in (67) and (68) to calculate a new gap profile, $h_{\text {new }}$. If $\int_{-10}^{10}\left(h_{\text {old }}-h_{\text {new }}\right)^{2} d x<10^{-5}$ then the calculation is stopped, else we iterate with $h_{\text {old }}=h_{\text {new }}$. For $\zeta=1$ the results are shown in figure 12bd. Not surprisingly, we find the same qualitative features discussed previously: $L$ has a maximum due to the competing effects of an increase in the gap thickness and the increased asymmetry of the contact zone.

\section{POROELASTIC LAYER}

Motivated in part by applications to the mechanics of cartilagenous joints, we now turn to the case of a cylinder moving above a fluid filled gel layer. This entails a different model for the constitutive behavior of the gel accounting for both the deformation of an elastic network and the fluid flowing through it. To describe the mechanical properties of a fluid filled gel we use poroelasticity, the continuum description of a material composed of an elastic solid skeleton and an interstitial fluid (Biot (1941); for a review of the literature see Cederbaum, Li \& Schulgasser (2000) or Wang (2000)). Our choice of poroelasticity to model the gel is motivated by the following scaling argument (Skotheim \& Mahadevan 2004a). Let $\nabla$ and $\nabla_{l_{p}}$ denote gradients on the system scale and the pore scale respectively; $p_{g}$ is the pressure varying on the system scale due to boundary conditions driving the flow, while $p_{p}$ is the pressure varying on the microscopic scale due to pore geometry. Fluid stress balance on the pore scale implies that the sum of the macroscopic pressure gradient driving the flow, $\nabla p_{g}$, and the microscopic pressure gradient, $\nabla_{l_{p}} p_{p}$, is balanced by the viscous resistance of the fluid having viscosity $\mu$ and velocity $\mathbf{v}, \mu \nabla_{l_{p}}^{2} \mathbf{v}$, so that the momentum balance in the fluid yields

$$
\mu \nabla_{l_{p}}^{2} \mathbf{v}-\nabla p_{g}-\nabla_{l_{p}} p_{p}=0
$$


When the pore scale, $l_{p}$, and system size, $H_{l}$, are well separated, i.e. $l_{p} / H_{l} \ll 1$, equation (177) yields the following scaling relations

$$
p_{g} \sim \frac{H_{l} \mu V}{l_{p}^{2}} \gg \frac{\mu V}{l_{p}} \sim p_{p},
$$

from which we conclude that the dominant contribution to the fluid stress tensor comes from the pressure. The simplest stress-strain law for the composite medium, proposed by Biot (1941), is found by considering the linear superposition of the dominant components of the fluid and solid stress tensor. If strains are small, the elastic behaviour of the solid skeleton is well characterized by isotropic Hookean elasticity. For a poroelastic material composed of a solid skeleton with Lamé coefficients $G$ and $\lambda$ when drained and a fluid volume fraction $\alpha$, the stress tensor $\boldsymbol{\sigma}$ is given by the constitutive equation

$$
\boldsymbol{\sigma}=G\left(\nabla \mathbf{u}+\nabla \mathbf{u}^{\mathrm{T}}\right)+\lambda \nabla \cdot \mathbf{u} \mathrm{I}-\alpha p_{g} \mathrm{I}
$$

The equations of equilibrium are

$$
\nabla \cdot \boldsymbol{\sigma}=0
$$

where we have neglected inertial effects. Mass conservation requires that the rate of dilatation of a solid skeleton having a bulk modulus $\beta^{-1}$ is balanced by the fluid entering the material element:

$$
\frac{k}{\mu} \nabla^{2} p_{g}=\beta \partial_{t} p_{g}+\partial_{t} \nabla \cdot \mathbf{u}
$$

$\beta \neq \lambda+2 G / 3$ since the Lamé coefficients $\lambda$ and $G$ are for the composite material and take into account the microstructure, while $\beta^{-1}$ is independent of the microstructure; for cartilage $\beta^{-1} \sim 1 \mathrm{GPa}$, while $G \sim \lambda \sim 1 \mathrm{MPa}$. Equations (79), (80) and (81) subject to appropriate boundary conditions describe the evolution of displacements $\mathbf{u}$ and fluid pressure $p_{g}$ in a poroelastic medium.

We now calculate the response of a poroelastic gel to an arbitrary time dependent pressure distribution before considering the specific case at hand. To make the equations dimensionless we use the following scalings

$$
\begin{gathered}
x=\sqrt{2 h_{0} R} x^{\prime}, \quad z=H_{l} z^{\prime}, \quad t=\tau t^{\prime}=\frac{\sqrt{2 h_{0} R}}{V-\omega R} t^{\prime}, \quad u_{z}=h_{0} u_{z}^{\prime}, \quad u_{x}=h_{0} u_{x}^{\prime} \\
p_{g}=p_{0} p_{g}^{\prime}=\mu(V-\omega R) \sqrt{\frac{2 R}{h_{0}^{3}}} p_{g}^{\prime}, \quad p=p_{0} p^{\prime}, \quad \boldsymbol{\sigma}=p_{0} \boldsymbol{\sigma}^{\prime}=\mu(V-\omega R) \sqrt{\frac{2 R}{h_{0}^{3}}} \boldsymbol{\sigma}^{\prime} .
\end{gathered}
$$


We take the thickness of the layer to be much smaller that the length scale of the contact zone, $\zeta=\frac{H_{l}}{\sqrt{2 h_{0} R}} \ll 1$, and consider a compressible material, $G \sim \lambda$. Then after dropping primes, the stress tensor (79) becomes

$$
\boldsymbol{\sigma}=\left(\begin{array}{cc}
\frac{1}{\eta} \frac{\lambda}{2 G+\lambda} \partial_{z} u_{z}+\frac{\zeta}{\eta} \partial_{x} u_{x}-\alpha p_{g} & \frac{1}{\eta} \frac{G}{2 G+\lambda} \partial_{z} u_{x}+\frac{\zeta}{\eta} \frac{G}{2 G+\lambda} \partial_{x} u_{z} \\
\frac{1}{\eta} \frac{G}{2 G+\lambda} \partial_{z} u_{x}+\frac{\zeta}{\eta} \frac{G}{2 G+\lambda} \partial_{x} u_{z} & \frac{1}{\eta} \partial_{z} u_{z}+\frac{\zeta}{\eta} \frac{\lambda}{2 G+\lambda} \partial_{x} u_{x}-\alpha p_{g}
\end{array}\right)
$$

Here,

$$
\eta=\frac{p_{0}}{2 G+\lambda} \frac{H_{l}}{h_{0}}=\sqrt{2} \frac{\mu(V-\omega R)}{2 G+\lambda} \frac{H_{l} R^{1 / 2}}{h_{0}^{5 / 2}},
$$

is the dimensionless number governing the relative size of the surface deflection to the undeformed gap thickness, i.e. the material compliance. Stress balance (80) yields

$$
\begin{array}{r}
0=\partial_{z z} u_{x}+\zeta\left(\frac{G+\lambda}{G} \partial_{x z} u_{z}-\eta \alpha \frac{2 G+\lambda}{G} \partial_{x} p_{g}\right)+\zeta^{2} \frac{2 G+\lambda}{G} \partial_{x x} u_{x} \\
0=\partial_{z z} u_{z}-\eta \alpha \partial_{z} p_{g}+\zeta \frac{G+\lambda}{2 G+\lambda} \partial_{x z} u_{x}+\zeta^{2} \frac{G}{2 G+\lambda} \partial_{x x} u_{z}
\end{array}
$$

and continuity (81) yields

$$
\frac{k \tau}{\mu H_{l}^{2} \beta}\left(\partial_{z z} p_{g}+\zeta^{2} \partial_{x x} p_{g}\right)=\partial_{t} p_{g}+\frac{h_{0}}{\beta H_{l} p_{0}} \partial_{t}\left(\partial_{z} u_{z}+\zeta \partial_{x} u_{x}\right) .
$$

To leading order (85) and (86) reduce to

$$
\begin{array}{r}
\partial_{z z} u_{x}=0 \\
\partial_{z z} u_{z}-\alpha \eta \partial_{z} p_{g}=0
\end{array}
$$

and

$$
\frac{k \tau}{\mu H_{l}^{2} \beta} \partial_{z z} p_{g}=\partial_{t} p_{g}+\frac{h_{0}}{\beta H_{l} p_{0}} \partial_{t z} u_{z}
$$

The normal unit vector to the soft interface is $\mathbf{n}=\left(-\left.\partial_{x} u_{z}\right|_{z=0}, 1\right)$, which in dimensionless form is

$$
\mathbf{n}=\left(-\left.\varepsilon \partial_{x} u_{z}\right|_{z=0}, 1\right)
$$

where $\varepsilon=\sqrt{\frac{h_{0}}{2 R}}$. The balance of normal traction on the solid-fluid interface yields

$$
\left.\boldsymbol{\sigma}_{f} \cdot \mathbf{n}\right|_{z=0}=\left.\boldsymbol{\sigma}_{s} \cdot \mathbf{n}\right|_{z=0}
$$

so that

$$
\begin{array}{r}
\left.\partial_{z} u_{x}\right|_{z=0}=0 \\
\left.\partial_{z} u_{z}\right|_{z=0}-\left.\alpha \eta p_{g}\right|_{z=0}=-\eta p .
\end{array}
$$


At the interface between the soft film and the underlying rigid substrate the no slip condition yields

$$
u_{z}(x,-1)=0, \quad u_{x}(x,-1)=0 .
$$

Solving (87), (91) and (92) yields

$$
\begin{array}{r}
u_{x}=0 \\
-\eta p=-\alpha \eta p_{g}+\partial_{z} u_{z}
\end{array}
$$

To calculate the displacement at the surface $u_{z}(x, 0, t)$ we need to determine the fluid pressure in the gel $p_{g}$. Using (93) in (88) yields

$$
\partial_{t} p_{g}-\gamma \partial_{z z} p_{g}=\delta \partial_{t} p
$$

where

$$
\gamma=\frac{\tau}{\tau_{p}}=\frac{k \sqrt{2 R h_{0}}(2 G+\lambda)}{H_{l}^{2}(V-\omega R)[\beta(2 G+\lambda)+\alpha]} \quad \text { and } \quad \delta=\frac{1}{\beta(2 G+\lambda)+\alpha} \sim O(1) .
$$

Here $\tau=\frac{l_{c}}{V} \sim \frac{\sqrt{h_{0} R}}{V}$ is the time scale associated with motion over the contact length, $\tau_{p} \sim \frac{\mu H_{l}^{2}}{k G}$ is the time scale associated with stress relaxation via fluid flow across the thickness of the gel, and $\delta \sim \frac{1}{\alpha}$ the inverse of the fluid volume fraction. Equation (94) corresponds to the short time limit discussed by Barry and Holmes (2001). The boundary conditions for (94) are determined by the fact that at the solid-gel interface there is no fluid flux and at the fluid-gel boundary there is no pressure jump so that

$$
\partial_{z} p_{g}(x,-1, t)=0, \quad p_{g}(x, 0, t)=p
$$

where both conditions are a consequence of Darcy's law for flow through a porous medium.

Although there is flux through the gel-fluid interface, the Reynolds equation (9) for the fluid pressure will remain valid if the fluid flux through the gel is much less than the flux through the thin gap. A fluid of viscosity $\mu$ flows with a velocity $\mathbf{v}=\left(v_{x}, v_{z}\right)$ through a porous medium of isotropic permeability $k$ according to Darcy's law, so that $\mathbf{v} \sim \frac{k}{\mu} \nabla p$. Hence, the total flux through a porous medium of thickness $H_{l}$ is $\int_{-H_{l}}^{0} v_{x} d z$, which will scale as $\frac{H_{l} k p_{0}}{\mu \sqrt{h_{0} R}} \sim \frac{H_{l} k V}{\mu h_{0}^{2}}$. Comparing this with the flux through the thin gap $h_{0} V$ leads to the dimensionless group $Q_{R}=\frac{H_{l} k}{h_{0}^{3}}$. If $Q_{R} \ll 1$ we can neglect the flow through the porous medium. For cartilage, $H_{l} \sim 1 \mathrm{~mm}$ and $k \sim 10^{-14} \mathrm{~mm}^{2}$, and flow through the porous medium 
can be neglected if $h_{0} \gg 10 \mathrm{~nm}$. This implies that the Reynolds lubrication approximation embodied in (9) remains valid in the gap for situations of biological interest.

In response to forcing, a poroelastic material can behave in three different ways depending on the relative magnitude of the time scale of the motion $\tau=l_{c} / V$ and the poroelastic time scale $\tau_{p}$. If $\tau \gg \tau_{p}$ the fluid in the gel is always in equilibrium with the surrounding fluid and a purely elastic theory for the deformation of the gel suffices; if $\tau \sim \tau_{p}$ the gel will behave as a material with a 'memory'; if $\tau \ll \tau_{p}$ the fluid has no time to move relative to the matrix and the poroelastic material will again behave as a solid albeit with a higher elastic modulus. In the physiological case of a cartilage layer in a rotational joint the poroelastic time scale for bovine articular cartilage is reported to be $\tau_{p} \approx 20$ seconds by Grodzinsky, Lipshitz \& Glimcher (1978), and $\tau_{p} \approx 500$ seconds by Mow, Holmes \& Lai (1984). For time scales on the order of 1 second, the cartilage should behave as a solid, but with an elastic modulus greater then that measured by equilibrium studies. We consider three different cases corresponding to:

\section{A. Low speed: $\tau_{m} \gg \tau_{p}$}

When the cylinder moves slowly, $\tau_{m} \gg \tau_{p}$, the time scale of the motion is much larger than the time scale over which the pressure diffuses $(i . e . \gamma \gg 1 \sim \delta$ ) so that (94) becomes

$$
\partial_{z z} p_{g}=0
$$

Solving (97) subject to (96) yields

$$
p_{g}=p
$$

i.e. at low speeds the fluid pressure in the gel is the same as the fluid pressure outside the gel. Equations (92) and (93) can be solved to yield

$$
u_{z}(x, 0, t)=-(1-\alpha) \eta p(x, t)
$$

We see that this limit gives a local relationship between the displacement of the gel surface and the fluid pressure in the gap, exactly as in the case of a purely elastic layer treated in 乌III 


\section{B. High speed: $\tau_{m} \ll \tau_{p}$}

When the time scale of the motion is much smaller than the time scale over which the pressure diffuses, i.e. $\gamma \ll 1 \sim \delta$, (94) becomes

$$
\partial_{t} p_{g}=\delta \partial_{t} p
$$

Since the gel is at equilibrium with the external fluid before the cylinder passes over it, $p_{g}(x, z,-\infty)=p(x,-\infty)=0$, and equation (100) yields

$$
p_{g}(x, z, t)=\delta p(x, t)
$$

Inserting (101) into (93) and integrating yields

$$
u_{z}(x, 0, t)=\frac{-\beta(2 G+\lambda) \eta}{\beta(2 G+\lambda)+\alpha} p(x, 1, t) .
$$

In this limit the fluid has no time to flow through the pores and the only compression is due to bulk compressibility of the composite gel, which now behaves much more rigidly. The effective elastic modulus of the solid layer is now $G_{\text {eff }} \sim \beta^{-1} \sim 1 \mathrm{GPa}$ rather than $G_{\text {eff }} \sim 1 \mathrm{MPa}$. However, the relationship between the pressure and displacement remains local as in $\oiiint$ III

We note that if $\nabla \cdot \mathbf{u}=0$, (81) has no forcing term and $p_{g}=0$. Poroelastic theory does not take into account shear deformations since these involve no local change in fluid volume fraction in the gel. In this case all the load will be borne by the elastic skeleton. However, shear deformation in a thin layer will involve geometric stiffening due to incompressibility so that the effective modulus will be $G_{e f f} \sim \frac{h_{0} R}{H_{l}^{2}} G$ (Skotheim \& Mahadevan 2004b). Hence, if $\frac{h_{0} R}{H_{l}^{2}} G \beta \ll 1$ the deformation should be treated as an incompressible layer as in $\$$ VI If $\frac{h_{0} R}{H_{l}^{2}} G \beta \gg 1$, the layer should be treated as in $\oiiint$ III with an effective modulus $\beta^{-1}$.

\section{Intermediate speeds: $\tau_{m} \sim \tau_{p}$}

When $\delta \sim \gamma \sim 1$ rewriting (94) for the difference between the fluid pressure inside and outside the gel, $p_{g}(x, z, t)-p(x, t)$, yields

$$
\partial_{t}\left(p_{g}-p\right)-\gamma \partial_{z z}\left(p_{g}-p\right)=(\delta-1) \partial_{t} p
$$


with the boundary conditions

$$
\partial_{z}\left(p_{g}-p\right)(x,-1, t)=0, \quad\left(p_{g}-p\right)(x, 0, t)=0 .
$$

We expand $p_{g}-p$ in terms of the solution of the homogeneous part of (103)-(104):

$$
p_{g}-p=\sum_{n=0}^{\infty} A_{n}(t) \sin \pi\left(n+\frac{1}{2}\right) z .
$$

Inserting the expansion into (103) we find

$$
\sum_{n=0}\left[\partial_{t} A_{n}+\gamma \pi^{2}\left(n+\frac{1}{2}\right)^{2} A_{n}\right] \sin \pi\left(n+\frac{1}{2}\right) z=(\delta-1) \partial_{t} p
$$

Multiplying (106) with $\sin \pi\left(m+\frac{1}{2}\right) z$ and integrating over the thickness yields

$$
\partial_{t} A_{n}+\gamma \pi^{2}\left(n+\frac{1}{2}\right)^{2} A_{n}=\frac{2(1-\delta)}{\pi\left(n+\frac{1}{2}\right)} \partial_{t} p
$$

Solving (107) for $A_{n}(t)$ yields

$$
A_{n}(t)=\frac{2(1-\delta)}{\pi\left(n+\frac{1}{2}\right)} \int_{-\infty}^{t} e^{-\gamma \pi^{2}\left(n+\frac{1}{2}\right)^{2}\left(t-t^{\prime}\right)} \partial_{t^{\prime}} p d t^{\prime} .
$$

Substituting (108) into (105) yields the fluid pressure in the gel

$$
p_{g}=p+\sum_{n=0} \frac{2(1-\delta) \sin \pi\left(n+\frac{1}{2}\right) z}{\pi\left(n+\frac{1}{2}\right)} \int_{-\infty}^{t} e^{-\gamma \pi^{2}\left(n+\frac{1}{2}\right)^{2}\left(t-t^{\prime}\right)} \partial_{t^{\prime}} p d t^{\prime} .
$$

Finally, (92), (93) and (109) yield

$$
u_{z}(1)=\eta\left[(-1+\alpha) p+\alpha \sum_{n=0} \frac{2(\delta-1)}{\pi^{2}\left(n+\frac{1}{2}\right)^{2}} \int_{-\infty}^{t} e^{-\gamma \pi^{2}\left(n+\frac{1}{2}\right)^{2}\left(t-t^{\prime}\right)} \partial_{t^{\prime}} p d t^{\prime}\right] .
$$

Since the higher order diffusive modes $(n>0)$ decay more rapidly than the leading order diffusive mode $(n=0)$, a good approximation to (110) is

$$
-\eta H(x)=u_{z}(0)=\eta\left[(-1+\alpha) p+\alpha \frac{8(\delta-1)}{\pi^{2}} \int_{-\infty}^{t} e^{\frac{-\gamma \pi^{2}}{4}\left(t-t^{\prime}\right)} \partial_{t^{\prime}} p d t^{\prime}\right] .
$$

This approximation is similar to that used in Skotheim \& Mahadevan (2004a). To simplify (111) for the case of interest we define

$$
\xi=\int_{-\infty}^{t} e^{\frac{-\gamma \pi^{2}}{4}\left(t-t^{\prime}\right)} \partial_{t^{\prime}} p d t^{\prime}
$$


so that

$$
\partial_{t} \xi=-\frac{\gamma \pi^{2}}{4} \xi+\partial_{t} p
$$

In the reference frame of the steadily moving cylinder $\xi(x, t)=\xi(x-t)$ so that

$$
\partial_{x} \xi=\frac{\gamma \pi^{2}}{4} \xi+\partial_{x} p
$$

Integrating the above yields

$$
\xi=-\int_{x}^{\infty} e^{\frac{-\gamma \pi^{2}}{4}\left(x^{\prime}-x\right)} \partial_{x^{\prime}} p d x^{\prime}
$$

Consequently, the distance between the gel and cylinder is found from (111), (111), (112) and (115) to be

$$
h(x)=1+x^{2}+\eta(1-\alpha)\left[p+\frac{8}{\pi^{2}} \int_{x}^{\infty} e^{\frac{-\gamma \pi^{2}}{4}\left(x^{\prime}-x\right)} \partial_{x^{\prime}} p d x^{\prime}\right]
$$

where

$$
\eta=\frac{p_{0} H_{l}}{(2 G+\lambda) h_{0}}=\frac{\sqrt{2 R} H_{l} \mu(V-\omega R)}{h_{0}^{5 / 2}(2 G+\lambda)},
$$

where we are considering the case where the bulk modulus of the skeletal material is much larger than the modulus of the elastic matrix $\beta G \ll 1$, so that (95) implies $\delta \approx \frac{1}{\alpha}$. This leaves us a system of equations (91), (10) and (116) for the pressure with 2 parameters: $\eta$ characterizes the deformation (softness); and $\gamma$ is the ratio of translational to diffusive timescales. The two limits $\gamma \ll 1$ and $\gamma \gg 1$ of (116) can both be treated using asymptotic methods. For $\gamma \gg 1$, (116) yields

$$
h=1+x^{2}+\eta(1-\alpha) p
$$

and we recover the limit of a thin compressible elastic layer treated in $₫$ with $\eta \rightarrow(1-\alpha) \eta$. For $\gamma \ll 1$, (116) yields

$$
h=1+x^{2}+\left(1-\frac{8}{\pi^{2}}\right)(1-\alpha) \eta p,
$$

which is the result for a thin compressible layer with $\eta \rightarrow\left(1-\frac{8}{\pi^{2}}\right)(1-\alpha) \eta$. When $\eta \ll \gamma \ll 1$ we expand the pressure field as in (26) writing $p=p_{0}+\eta p_{1}$, where $p_{0}=\frac{2 x}{\left(1+x^{2}\right)^{2}}$ as in (31). Inserting this expression into (116) yields

$$
h=1+x^{2}+\eta(1-\alpha)\left\{p_{0}+\frac{8}{\pi^{2}} \int_{x}^{\infty}\left[1+\frac{\pi^{2} \gamma}{4}\left(x-x^{\prime}\right)\right] \partial_{x^{\prime}} p_{0} d x^{\prime}\right\}+O(\gamma \eta)
$$

which can be integrated to give

$$
h=1+x^{2}+\eta(1-\alpha)\left[\frac{\left(\pi^{2}-8\right) 2 x}{\pi^{2}\left(1+x^{2}\right)^{2}}+\frac{2 \gamma}{\left(1+x^{2}\right)}\right] .
$$


We see that increasing $\gamma$ increases the gap thickness and lowers the pressure without increasing the asymmetry, thus decreasing the lift. In the small deflection limit, $\eta \ll 1$, the dimensionless lift force is $L=C_{p}(\gamma) \eta$ and the lift force in dimensional terms is

$$
L=C_{p}(\gamma)(1-\alpha) \frac{\mu^{2} V^{2}}{2 G+\lambda} \frac{H_{l} R^{3 / 2}}{h_{0}^{7 / 2}}
$$

where $C_{p}$ is a function of $\gamma$ and shown graphically in figure [13].

When $\eta=O(1)$, we use a numerical method to solve (9), (10) and (116) on a finite domain using the continuation software AUTO (Doedel et al. 2004) with $\eta$ and $\gamma$ as the continuation parameters. The initial solution from which the continuation begins is with

$\eta=\gamma=0$, corresponding to $h=1+x^{2}$, and $p=\frac{2 x}{\left(1+x^{2}\right)^{2}}$. The form of the lift force as a function of $\eta, L(\eta, \gamma)$ for various $\gamma$ can be almost perfectly collapsed onto a single curve after appropriately scaling the $\eta, L$ axes using the position of the maximum; i.e. $\frac{L\left[\eta / \eta_{\max }(\gamma)\right]}{L_{\max }(\gamma)}$ where $L_{\max }$ and $\eta_{\max }$ are shown in figure $13 \mathrm{~b}, \mathrm{c}$.

\section{ELASTIC SHELL}

For elastic layers attached to a rigid substrate the effective stiffness increases with decreasing thickness. However, for free elastic shells the effective stiffness increases with increasing thickness. To see the effects of this type of geometry in biolubrication problems, we turn our attention to a configuration in which a surface is rendered soft through its geometry rather than its elastic moduli. We consider a half-cylindrical elastic shell of thickness $h_{s}$ and radius $R$ moving with constant velocity $V$ parallel to the rigid substrate, while completely immersed in fluid of viscosity $\mu$, as shown in figure 14. The shell is clamped at its edges, which are at a height $R+h_{0}$ above the rigid solid. The shape of the elastic half-cylinder is governed by the elastica equation (for the history of the elastica equation as well as its derivation see Love 1944) for $\theta(s)$, the angle between the horizontal and the tangent vector where $s$ is the arc length coordinate. Balancing torques about the point $O$ in figure 15 gives: $M(s+d s)-M(s)-d s n_{x} \sin \theta+d s n_{z} \cos \theta+\frac{d s^{2}}{2}\left(p \cos \theta-\mu \partial_{z} u \sin \theta-p \partial_{s} h \sin \theta\right)=$ 0. In the limit $d s \rightarrow 0, \partial_{x} M-n_{x} \sin \theta+n_{z} \cos \theta=0$. The $x$-force balance is $n_{x}(s+d s)-n_{x}(s)-d s\left(p \partial_{s} h+\mu \partial_{x} u\right)=0$, which as $d s \rightarrow 0$ yields $\partial_{s} n_{x}=\mu \partial_{x} u+p \partial_{s} h$. The $z$-force balance is $n_{z}(s+d s)-n_{z}(s)+p d s=0$, which as $d s \rightarrow 0$ yields $\partial_{s} n_{z}=-p$. We note that external forces are applied in the contact region where derivatives taken with respect 
to $x$ are interchangeable with those taken with respect to $s$, i.e. $\partial_{x} h \approx \partial_{s} h$ and $\partial_{x} p \approx \partial_{s} p$, which allows for a consistent framework for the fluid and solid equilibrium equations. This yields

$$
\frac{G(\lambda+G) h_{s}^{3}}{3(\lambda+2 \mu)} \partial_{s s} \theta=n_{x} \sin \theta-n_{z} \cos \theta
$$

where the stress resultants $n_{x}, n_{z}$ are determined by the equations

$$
0=\partial_{s} n_{x}+\mathbf{e}_{\mathbf{x}} \cdot \boldsymbol{\sigma}_{f} \cdot \mathbf{n}, \quad 0=\partial_{s} n_{z}+\mathbf{e}_{\mathbf{z}} \cdot \boldsymbol{\sigma}_{f} \cdot \mathbf{n}
$$

i.e.

$$
\partial_{s} n_{x}=\frac{\mu V}{h}+\frac{h \partial_{s} p}{2}+p \partial_{s} h, \quad \partial_{s} n_{z}=-p
$$

where, $\mathbf{n}=\left(\partial_{s} h,-1\right)$, and $\boldsymbol{\sigma}_{f}$ is given by (2). Then, the pressure in the fluid is governed by the Reynolds equation (9)

$$
\partial_{s s} p=\frac{-\partial_{s} h}{h^{3}}\left(6 \mu V+3 h^{2} \partial_{s} p\right)
$$

Finally, since cylindrical deformations are inextensional, we must complement (123)-(126) with the kinematic equations

$$
\partial_{s} X=\cos \theta, \quad \partial_{s} h=\sin \theta .
$$

where the position of the surface of the elastic cylinder is $(X, h)$. The equations (123)-(127) are made dimensionless with the following scalings

$$
\begin{gathered}
p=p_{0} p^{\prime}=\frac{\sqrt{2} \mu V R^{1 / 2}}{h_{0}^{3 / 2}} p^{\prime}, \quad s=\pi R s^{\prime}, \quad h=R Z, \\
X=R X^{\prime} \quad n_{x}=p_{0} h_{0} n_{x}^{\prime}, \quad n_{z}=p_{0} \sqrt{2 h_{0} R} n_{z}^{\prime} .
\end{gathered}
$$

After dropping primes, the dimensionless forms of equations (123)-(127) are written as

$$
\begin{aligned}
\partial_{s s} p & =\frac{-\partial_{s} Z}{Z^{3}}\left[3 \sqrt{2} \pi\left(\frac{h_{0}}{R}\right)^{3 / 2}+3 Z^{2} \partial_{s} p\right], \\
\partial_{s} n_{x} & =\frac{R}{h_{0}}\left(p \partial_{s} Z+\frac{Z \partial_{s} p}{2}\right)+\sqrt{\frac{h_{0}}{2 R}} \frac{\pi}{Z}, \\
\partial_{s} n_{z} & =-\pi \sqrt{\frac{R}{2 h_{0}}} p \\
\partial_{s} X & =\pi \cos \theta \\
\partial_{s} Z & =\pi \sin \theta \\
\partial_{s s} \theta & =3 \sqrt{2} \pi^{2} \frac{\mu V(\lambda+2 G)}{G(\lambda+G)} \frac{R^{5 / 2}}{h_{s}^{3} h_{o}^{1 / 2}}\left(n_{x} \sin \theta-\sqrt{\frac{2 R}{h_{0}}} n_{z} \cos \theta\right)
\end{aligned}
$$


To find the scale of the elastic deformation $\eta=\frac{H_{0}}{h_{0}}$, where the maximum displacement of the cylinder is of order $H_{0}$, we note that the change in curvature is of order $\frac{H_{0}}{R^{2}}$ so that the bending strain is $\epsilon=\frac{h_{s} H_{0}}{R^{2}}$ and the elastic energy per unit length therefore scales as $\int G \epsilon^{2} d A \sim \int G\left(\frac{h_{s} H_{0}}{R^{2}}\right)^{2} d A \sim \frac{G h_{s}^{3} H_{0}^{2}}{R^{3}}$. The work done by the fluid is due to a localized torque and scales as $\int p x d x \sim p_{0} h_{0} R$, acting through an angle $\Delta \theta \sim \frac{H_{0}}{R}$. Balancing the work done by the fluid torque $p_{0} h_{0} H_{0}$ with the elastic energy $\frac{G h_{s}^{3} H_{0}^{2}}{R^{3}}$ yields

$$
\eta \sim \frac{H_{0}}{h_{0}} \sim \frac{\mu V}{G} \frac{R^{7 / 2}}{h_{s}^{3} h_{0}^{3 / 2}}
$$

so that (134) can be written as

$$
\partial_{s s} \theta=\frac{h_{0} \eta}{R}\left(n_{x} \sin \theta-\sqrt{\frac{2 R}{h_{0}}} n_{z} \cos \theta\right) .
$$

The system (129)-(133), (136) for $\theta, p, n_{x}, n_{z}, x, z$ has two dimensionless parameters, $\eta=$ $3 \sqrt{2} \pi^{2} \frac{\mu V(\lambda+2 G)}{G(\lambda+G)} \frac{R^{7 / 2}}{h_{s}^{3} h_{0}^{3 / 2}}$ and $\frac{h_{0}}{R}$, and is subject to 8 boundary conditions

$$
\begin{aligned}
& p(0)=p(1)=0 \\
& X(0)=-1, \quad X(1)=1 \\
& Z(0)=Z(1)=1+\frac{h_{0}}{R}, \\
& \theta(0)=\frac{-\pi}{2}, \quad \theta(1)=\frac{\pi}{2} .
\end{aligned}
$$

The first two are a consequence of lubrication theory, while the rest are kinematic boundary conditions on the cylinder's lateral edges, which are assumed to be clamped. We note that if the cylinder has a natural curvature this will not effect the system of equations (123)-(127), but may change the boundary conditions.

We solve (129)-(133), (136) and (137) numerically using the continuation software AUTO 2000 (Doedel et al. 2004) with $\eta$ as the continuation parameter. For $\eta<1$, the dimensionless lift $L=C_{s} \eta$, where the constant $C_{s}\left(\frac{h_{0}}{R}\right)$ is shown in figure 17b. However, we see that even for $\eta<100$ the linear relationship remains valid. The dimensional lift force for $\eta<100$ is

$$
L=6 \sqrt{2} \pi^{2} \frac{\mu^{2} V^{2}(\lambda+2 G)}{G(\lambda+G)} \frac{R^{9 / 2}}{h_{s}^{3} h_{0}^{5 / 2}} C_{s}\left(\frac{h_{0}}{R}\right) .
$$

Figure 16] shows the gap thickness profile and pressure distribution and figure [17 shows the dimensionless lift for $\frac{h_{0}}{R}=0.001$. As $\eta$ increases the elastic deformation breaks the symmetry of the gap thickness profile and results in an asymmetric pressure distribution 
and corresponding lift. However, the concomitant increase in gap thickness decreases the magnitude of the pressure. As for the previous systems considered, the competition between symmetry breaking (dominant for small $\eta$ ) and decreasing pressure (dominant for large $\eta$ ) produces a maximum in the lift. The form of the lift force as a function of $\eta, L\left(\eta, \frac{h_{0}}{R}\right)$ for various $\frac{h_{0}}{R}$ can be almost perfectly collapsed onto a single curve after appropriately scaling the $\eta, L$ axes; i.e. $\frac{L\left[\eta / \eta_{\max }\left(\frac{h_{0}}{R}\right)\right]}{L_{\max }\left(\frac{h_{0}}{R}\right)}$ where $L_{\max }$ and $\eta_{\max }$ are shown in figure 17k, $\mathrm{d}$.

\section{JOURNAL BEARING}

So far, with the exception of $₫ \mathbb{I V}$ we have dealt only with non-conforming contacts. In this section we consider an elastohydrodynamic journal bearing: a geometry consisting of a cylinder rotating within a larger cylinder that is coated with a soft solid. The journal bearing is a conforming contact and is a better representation of bio-lubrication in mammalian joints in which synovial fluid lubricates bone coated with thin soft cartilage layers. Previous analyses of the elastohydrodynamic journal bearing have focused on situations where fluid cavitation needs to be taken into account (O'Donoghue, Brighton \& Hooke 1967; Conway \& Lee 1975). As before, we restrict our attention the case where the surface deforms appreciably before the cavitation threshold is reached so that the gap remains fully flooded. A schematic diagram is shown in figure 18

We take the center of the inner cylinder, $O_{i}$, to be the origin; the center of the outer cylinder, $O_{o}$, is located at $x=\epsilon_{x}, z=\epsilon_{z}$. The inner cylinder of radius $R$ rotates with angular velocity $\omega$; the stationary outer cylinder of radius $R+h_{0}+H_{l}$ is coated with a soft solid of thickness $H_{l} \ll R$ and Lamé coefficients $\lambda$ and $G$. Here, $h_{0}$ is the average distance between the inner cylinder and the soft solid. Following Leal (1992), we use cartesian coordinates to describe the eccentric geometry and applied forces, but use polar coordinates to describe the

fluid motion. When $\frac{h_{0}}{R} \ll 1$, the lubrication approximation reduces the Stokes equations in a cylindrical geometry for a fluid of viscosity $\mu$, pressure $p$, and velocity field $\mathbf{v}=\left(v_{r}, v_{\theta}\right)$, to (Leal 1992)

$$
\begin{array}{r}
\partial_{r} p=0, \\
\frac{1}{R} \partial_{\theta} p=\mu \partial_{r r} v_{\theta},
\end{array}
$$


subject to the boundary conditions

$$
\begin{array}{r}
v_{r}=0, \quad v_{\theta}=-\omega R \text { at } r=R, \\
v_{r}=0, \quad v_{\theta}=0 \text { at } r=R+h, \\
p(0)=p(2 \pi) .
\end{array}
$$

Since $\frac{h_{0}}{R} \ll 1$, the continuity equation simplifies to (Leal 1992)

$$
R \partial_{r} v_{r}+\partial_{\theta} v_{\theta}=0
$$

and the gap thickness profile simplifies to

$$
h(\theta)=h_{0}+\epsilon_{x} \cos \theta+\epsilon_{z} \sin \theta+H(\theta)
$$

where $H(\theta)$ is the elastic interface displacement due to the fluid forces. As in $₫$ III

$$
H(\theta)=\frac{H_{l} p(\theta)}{2 G+\lambda}
$$

so that (142) yields

$$
h=h_{0}+\epsilon_{x} \cos \theta+\epsilon_{z} \sin \theta+\frac{H_{l} p}{2 G+\lambda} .
$$

Using the following primed dimensionless variables,

$$
\begin{array}{r}
v_{r}=\omega h_{0} v_{r}^{\prime}, \quad v_{\theta}=\omega R v_{\theta}^{\prime}, \quad r=h_{0} r^{\prime}, \quad h=h_{0} h^{\prime}, \\
p=p^{*} p^{\prime}=\frac{\mu R^{2} \omega}{h_{0}^{2}} p^{\prime}, \quad \epsilon_{x}=h_{0} \epsilon_{x}^{\prime}, \quad \epsilon_{z}=h_{0} \epsilon_{z}^{\prime},
\end{array}
$$

we write (139)-(141), (144), after dropping the primes, as

$$
\begin{array}{r}
\partial_{r} p=0, \\
\partial_{\theta} p=\partial_{r r} v_{\theta}, \\
\partial_{r} v_{r}+\partial_{\theta} v_{\theta}=0, \\
h=1+\epsilon_{x} \cos \theta+\epsilon_{z} \sin \theta+\eta p,
\end{array}
$$

subject to

$$
\begin{array}{r}
v_{r}=0, \quad v_{\theta}=-1 \text { at } r=\frac{R}{h_{0}}, \\
v_{r}=0, \quad v_{\theta}=0 \text { at } r=\frac{R}{h_{0}}+h, \\
p(0)=p(2 \pi),
\end{array}
$$


where,

$$
\eta=\frac{H_{l} p^{*}}{h_{0}(2 G+\lambda)}=\frac{\mu \omega R^{2} H_{l}}{(2 G+\lambda) h_{0}^{3}},
$$

is the softness parameter. As in $\$$ w we use (146) to derive the system of equations for the fluid pressure

$$
\begin{array}{r}
\partial_{\theta}\left(6 h+h^{3} \partial_{\theta} p\right)=0, \\
h=1+\epsilon_{x} \cos \theta+\epsilon_{z} \sin \theta+\eta p, \\
p(0)=p(2 \pi) .
\end{array}
$$

In addition, fluid incompressibility implies that the average deflection must vanish:

$$
\int_{0}^{2 \pi} h d \theta=2 \pi \rightarrow \int_{0}^{2 \pi} p d \theta=0 .
$$

The forces on the inner cylinder are

$$
\int_{0}^{2 \pi} p \sin \theta d \theta=L_{z}, \quad \int_{0}^{2 \pi} p \cos \theta d \theta=L_{x}
$$

Here, $L_{z}$ is the vertical force and $L_{x}$ is the horizontal force. We begin with the classical solution for a rigid journal bearing (Leal 1992): $L_{z}=1, L_{x}=\eta=\epsilon_{z}=0, \epsilon_{x}=0.053$. The following brief symmetry argument shows that $\epsilon_{z}=0$ when $\eta=L_{x}=0$. Since Stokes equations for viscous flow are reversible in time, the transformation $\omega \rightarrow-\omega$ implies that $\epsilon_{z} \rightarrow-\epsilon_{z}$. However, due to symmetry we expect the solution to be a reflection about the $z$-axis and we conclude that $\epsilon_{z}=0$.

As in previous sections we investigate how elastohydrodynamics alters this picture by specifying the eccentricity $\left(\epsilon_{x}, \epsilon_{z}\right)$ and calculating the forces $\left(L_{x}, L_{z}\right)$ as a function of the softness parameter $\eta$. Solutions to (149)-(151) are computed numerically using the continuation software AUTO 2000 (Doedel et al. 2004) with $\eta$ as the continuation parameter and the solution for $\eta=0$ as the initial guess. Just as for different geometries analyzed in previous sections, the deflection of the surface of the soft solid breaks the symmetry and leads to the generation of a horizontal force in the $x$-direction: $L_{x}>0$. For small deformations $(\eta \ll 1)$ the dimensionless horizontal force $L=C_{j}\left(\epsilon_{x}\right) \eta$, where the coefficient $C_{j}\left(\epsilon_{x}\right)$ is shown in figure 19, In dimensional terms, the horizontal force per unit length for small deformations is

$$
L_{x}=C_{j}\left(\epsilon_{x}\right) \frac{p^{*} H_{l}}{(2 G+\lambda) h_{0}} p^{*} R=C_{j}\left(\epsilon_{x}\right) \frac{\mu^{2} \omega^{2} R^{5} H_{l}}{(2 G+\lambda) h_{0}^{5}} .
$$


For nearly concentric cylinders, $\epsilon_{x} \ll 1, C_{j}\left(\epsilon_{x}\right)=115 \epsilon_{x}+O\left(\epsilon_{x}^{2}\right)$. For large eccentricities, $\epsilon_{x} \rightarrow 1$, the lubrication pressure diverges and $C_{j}\left(\epsilon_{x}\right) \approx 12.3\left(1-\epsilon_{x}\right)^{-3}$. For $\eta \nless 1$ we show $L, h$ and $p$ in figure 20 .

\section{3-DIMENSIONAL LUBRICATION FLOW}

The analysis of the 3-dimensional problem of a sphere moving close to a soft substrate is considerably more involved. Stone et al. (in preparation) are currently engaged in using perturbation methods to calculate the elastohydrodynamic lift for the case of a sphere translating above a thin elastic layer. Here, we restrict ourselves to the use scaling arguments to generalize the quantitative results of previous sections to spherical sliders. The results are tabulated in Table 2. In the fluid layer separating the solids, balancing the pressure gradient with the viscous stresses yields

$$
\frac{p}{l_{c}} \sim \frac{\mu V}{h^{2}} \rightarrow p \sim \frac{\mu V l_{c}}{h^{2}}
$$

where $l_{c}$ is the size of the contact zone. Substituting $h=h_{0}+H_{0}$ with $H_{0} \ll h_{0}$ we find that the lubrication pressure is

$$
p \sim \frac{\mu V l_{c}}{\left(h_{0}+H_{0}\right)^{2}} \sim \frac{\mu V l_{c}}{h_{0}^{2}}\left(1+\frac{h_{0}}{R}\right)=\frac{\mu V l_{c}}{h_{0}^{2}}(1+\eta) .
$$

The reversibility of Stokes equations and the symmetry of paraboloidal contacts implies that

the lift force $L=0$ when $\eta=\frac{H_{0}}{h_{0}}=0$. For $\eta \ll 1$, we expand the pressure as $p=p^{(0)}+\eta p^{(1)}$. Since $p=p^{(0)}$ will not generate vertical forces, the the lift on a spherical slider, $L_{s}$, will scale as

$$
L_{s}=\eta \int p^{(1)} d A \sim \eta \int \frac{\mu V l_{c}}{h_{0}^{2}} d A \sim \eta \frac{\mu V l_{c}^{3}}{h_{0}^{2}} .
$$

To compute $L$, we need a prescription for the softness $\eta$ and the contact radius $l_{c}$ for each configuration.

(A) For a thin compressible elastic layer (乌III), we substitute $l_{c} \sim \sqrt{h_{0} R}$ and $\eta \sim$ $\frac{\mu(V-\omega R)}{2 G+\lambda} \frac{H_{l} R^{1 / 2}}{h_{0}^{5 / 2}}$ into (155) to find

$$
L_{s} \sim \frac{\mu^{2} V^{2} H_{l} R^{2}}{h_{0}^{3}(2 G+\lambda)} .
$$


(B) For a thin elastic layer with a degenerate axisymmetric conforming contact (乌IV), $l_{c} \sim$ $\left(h_{0} R^{2 n-1}\right)^{1 / 2 n}$ and $\eta \sim \frac{\mu V}{2 G+\lambda} \frac{H_{l} R^{1-\frac{1}{n}}}{h_{0}^{3-\frac{1}{2 n}}}$ so that (155) yields

$$
L_{s} \sim \frac{\mu^{2} V^{2} H_{l} R^{4-\frac{2}{n}} h_{0}^{-5+\frac{2}{n}}}{2 G+\lambda}
$$

(C) For a soft spherical slider (or thick layer $H_{l} \gg l_{c}$; $\$$ VD), the deflection is given by (45) so that

$$
H(x, y)=\frac{\lambda+2 G}{4 \pi G(\lambda+G)} \int \frac{p\left(x^{\prime}, y^{\prime}\right) d x^{\prime} d y^{\prime}}{\sqrt{\left(x^{\prime}-x\right)^{2}+\left(y^{\prime}-y\right)^{2}}} \sim \frac{p l_{c}}{G} .
$$

so that

$$
\eta \sim \frac{p l_{c}}{G h_{0}} .
$$

the size of the contact zone $l_{c} \sim \sqrt{h_{0} R}$ so that (155) and (159) yield

$$
L_{s} \sim \frac{\mu^{2} V^{2}}{G} \frac{R^{5 / 2}}{h_{0}^{5 / 2}}
$$

(D) For an incompressible layer ( $(\mathrm{DI})$ we have two cases depending on the thickness of the substrate relative to the contact zone characterized by the parameter $\zeta=\frac{H_{l}}{l_{c}}$. For $\zeta \gtrsim 1$, $l_{c} \sim \sqrt{h_{0} R}$ and $\eta \sim \frac{\mu(V-\omega R)}{G} \frac{R}{h_{0}^{2}}$ so that (155) yields

$$
L_{\zeta \gtrsim 1} \sim \frac{\mu^{2} V^{2}}{G} \frac{R^{5 / 2}}{h_{0}^{5 / 2}}
$$

For the case $\zeta \ll 1$ the proximity of the undeformed substrate substantially stiffens the layer. In sharp contrast to a compressible layer, a thin incompressible layer will deform via shear with an effective shear strain $\frac{\Delta u}{H_{l}}$. An incompressible solid must satisfy the continuity equation $\nabla \cdot \mathbf{u}=0$, which implies that $\frac{\Delta u}{l_{c}} \sim \frac{H_{0}}{H_{l}}$. Consequently, $\frac{\Delta u}{H_{l}} \sim \frac{l_{c} H_{0}}{H_{l}^{2}}$. Balancing the elastic energy $\int G\left(\frac{l_{c} H_{0}}{H_{l}^{2}}\right)^{2} d V \sim G\left(\frac{l_{c} H_{0}}{H_{l}^{2}}\right)^{2} H_{l} l_{c}^{2}$ with the work done by the pressure $p H_{0} l_{c}^{2}$ yields

$$
\eta \sim \frac{p}{G} \frac{H_{l}^{3}}{h_{0} l_{c}^{2}}
$$

Since $l_{c} \sim \sqrt{h_{0} R}$, (153), (155) and (162) yield

$$
\eta \sim \frac{\mu V}{G} \frac{H_{l}^{3}}{h_{0}^{7 / 2} R^{1 / 2}}, \quad L_{\zeta \ll 1} \sim \frac{\mu^{2} V^{2}}{G} \frac{H_{l}^{3} R}{h_{0}^{4}}
$$

(E) For a thin poroelastic layer ( $(\overline{V I I I}), l_{c} \sim \sqrt{h_{0} R}$ and $\eta \sim \frac{\mu(V-\omega R)}{2 G+\lambda} \frac{H_{l} R^{1 / 2}}{h_{0}^{5 / 2}}$ so that (155) yields

$$
L_{s} \sim C(\gamma) \frac{\mu^{2} V^{2}}{2 G+\lambda} \frac{H_{l} R^{2}}{h_{0}^{3}}
$$


where $\gamma$ is the ratio of the poroelastic time scale to the time scale of the motion. (F) For a spherical shell slider (\$VIII) there are two cases: the thickness of the shell, $h_{s}$, is smaller than the gap thickness, i.e. $h_{s} \ll h_{0}$ and all the elastic energy is stored in stretching; or $h_{s} \gtrsim h_{0}$ and bending and stretching energies are of the same order of magnitude (Landau \& Lifshitz 1970). For a localized force the deformation will be restricted to a region of area $d^{2}$. The stretching energy per unit area scales as $G h_{s} H_{0}^{2} / R^{2}$, while the bending energy scales as $G h_{s}^{3} H_{0}^{2} / d^{4}$. The total elastic energy, $U$, of the deformation is then given by

$$
U \approx \frac{G h_{s} H_{0}^{2} d^{2}}{R^{2}}+\frac{G h_{s}^{3} H_{0}^{2}}{d^{2}}
$$

which has a minimum at $d=\sqrt{h_{s} R}$. Comparing $d$ with $l_{c} \sim \sqrt{h_{0} R}$ we see that the hydrodynamic pressure is localized if $h_{s}>h_{0}$. For a localized force $d=\sqrt{h_{s} R}$ while for a non-localized force $d=R$. The elastic energy of a localized deformation, $U_{l}$, and a nonlocalized deformation, $U_{n}$, are given by

$$
U_{l}=\frac{G h_{s}^{2} H_{0}^{2}}{R}, \quad U_{n}=G h_{s} H_{0}^{2}
$$

The moment exerted by the hydrodynamic pressure on the spherical shell slider is

$$
M \sim p l_{c}^{3}
$$

which is independent of $h_{0}$. The work done by the moment (167), which acts through an angle $\Delta \theta \sim H_{0} / d$, is

$$
M \Delta \theta \sim p l_{c}^{3} \frac{H_{0}}{d} .
$$

Balancing the work done by the fluid (168) with the stored elastic energy (166) for both nonlocal and local deformations yields

$$
\eta_{n} \sim \frac{\mu V R}{G h_{s} h_{0}}, \quad \eta_{l} \sim \frac{\mu V R^{5 / 2}}{G h_{s}^{5 / 2} h_{0}},
$$

so that (155) and (169) yield the lift force on the sphere for the two cases

$$
\begin{array}{cc}
L_{l} \sim \frac{\mu^{2} V^{2}}{G} \frac{R^{4}}{h_{s}^{5 / 2} h_{0}^{3 / 2}} \quad \text { for } \frac{h_{s}}{h_{0}} \gg 1, \\
L_{n} \sim \frac{\mu^{2} V^{2}}{G} \frac{R^{5 / 2}}{h_{s} h_{0}^{3 / 2}} \quad \text { for } \frac{h_{s}}{h_{0}} \lesssim 1 .
\end{array}
$$

(G) For the ball and socket configuration, roughly the 3-dimensional analog of the journal bearing (乌IX), $l_{c} \sim R$ and $\eta \sim \frac{\mu \omega R^{2} H_{l}}{(2 G+\lambda) h_{0}^{3}}$ so that the horizontal force is given by (155):

$$
L_{s} \sim \frac{\mu \omega^{2} R^{2}}{2 G+\lambda} \frac{H_{l} R^{4}}{h_{0}^{5}} .
$$




\section{DISCUSSION}

The various combinations of geometry and material properties in this paper yield some simple results of great generality : the elastohydrodynamic interaction between soft surfaces immersed in a viscous fluid leads generically to a coupling between tangential and normal forces regardless of specific material properties or geometrical configurations, i.e. a lift force that arises due to the asymmetric fluid pressure deforming the soft solid which breaks the symmetry of the gap profile. For small surface deformations,

$\eta=\frac{\text { surface displacement }}{\text { characteristic gap thickness }} \ll 1$, the dimensionless normal force is linear in $\eta$. Increasing $\eta$ (i.e. softening the material) increases the asymmetry but decreases the magnitude of the pressure. The competition between symmetry breaking, which dominates for small $\eta$, and decreasing pressure, which dominates for large $\eta$, produces a maximum in the lift force as a function of $\eta$, the material's softness.

Additional complications such as nonlinearities and anisotropy in both the fluid and solid, streaming potentials and current generated stresses (Frank \& Grodzinsky 1987a,b) would clearly change some of our conclusions. However, the robust nature of the coupling between the tangential and normal forces illustrated in this paper should persist and suggests both experiments and design principles for soft lubrication.

\section{Acknowledgments}

The authors thank Mederic Argentina for assistance using the AUTO 2000 software package, and both Howard Stone and Tim Pedley for their thoughtful comments. We acknowledge support via the Norwegian Research Council (JS), the US Office of Naval Research Young Investigator Program (LM) and the US National Institutes of Health (LM).

[1] M. Abkarian, C. Lartigue \& A. Viallat, "Tank treading and unbinding of deformable vesicles in shear flow: determination of the lift force," Phys. Rev. Lett. 88, 068103 (2002).

[2] S.I. Barry \& M. Holmes, "Asymptotic behaviors of thin poroelastic layers," IMA Journal of Applied Mathematics 66, 175-194 (2001). 
[3] G.K. Batchelor An introduction to fluid dynamics (Cambridge University Press, Cambridge, UK, 1967).

[4] J. Beaucourt, T. Biben \& C. Misbah, "Optimal lift force on vesicles near a compressible substrate," Europhys. Lett. 67 676-682 (2004).

[5] M.A. Biot, "General Theory of Three-Dimensional Consolidation," Journal of Applied Physics 12, 155-165 (1941).

[6] G. Cederbaum, L.P. Li \& K. Schulgasser, Poroelastic structures (Elsevier, Oxford, 2000).

[7] H.D. Conway \& H.C. Lee "The analysis of the lubrication of a flexible journal bearing," Trans. ASME J. Lub. Tech. 97 599-604 (1975).

[8] E.R. Damiano, B.R. Duling, K. Ley \& T.C. Skalak, "Axisymmetric pressure-driven flow of rigid pellets through a cylindrical tube lined with a deformable porous wall layer," J. Fluid Mech. 314, 163-189 (1996).

[9] R. H. Davis, J-M Serayssol \& E. J. Hinch, "The elastohydrodynamic collision of two spheres," J. Fluid Mech. 163 479-497 (1986).

[10] E.J. Doedel, R.C. Paffenroth, A.R. Champneys, T.F. Fairfrieve, Y.A. Kuznetsov, B.E. Oldeman, B. Sandstede \& X. Wang, AUTO 2000: Continuation and Bifurcation Software for Ordinary Differential Equations (2004).

[11] D. Dowson \& G.R. Higginson, "A numerical solution to the elasto-hydrodynamic problem," J. Mech Eng. Sci. 1, 7-15 (1959).

[12] J. Feng \& S. Weinbaum, "Lubrication theory in highly compressible porous media: the mechanics of skiing, from red cells to humans," J. Fluid Mech. 422, 281-317 (2000).

[13] J.M. Fitz-Gerald, "Mechanics of red-cell motion through very narrow capillaries," Proc. Roy. Soc. Lond. B 174, 193-227 (1969).

[14] E.H. Frank \& A.J. Grodzinsky, "Cartilage electromechanics. I. Electrokinetic transduction and the effects of electrolyte pH and ionic strength," J. Biomech. 20, 615-27 (1987a)

[15] E.H. Frank \& A.J. Grodzinsky, "Cartilage electromechanics. II. A continuum model of cartilage electrokinetics and correlation with experiments," J. Biomech. 20, 629-39 (1987b)

[16] G.M.L. Gladwell, Contact Problems in the Classical Theory of Elasticity (Sijthoff and Noordhoff, Alphen aan den Rijn, The Netherlands, 1980)

[17] A.J. Grodzinsky, H. Lipshitz \& M.J. Glimcher, "Electromechanical properties of articular cartilage during compression and stress relaxation," Nature 275, 448-450 (1978). 
[18] B.J. Hamrock, Fundamentals of fluid film lubrication (McGraw-Hill, New York, 1994).

[19] B.J. Hamrock \& D. Dowson, Ball bearing lubrication: the elastohydrodynamics of elliptical contacts (John Wiley \& Sons, New York, 1981).

[20] J. Happel \& H. Brenner, Low reynolds number hydrodynamics (Kluwer Boston Inc., Hingham MA, 1983).

[21] D.J. Jeffrey \& Y. Onishi, "The slow motion of a cylinder next to a plane wall," Q. J. Appl. Math. 34 Pt. 2, 129-137 (1981).

[22] K.L. Johnson, Contact mechanics (Cambridge University Press, Cambridge UK, 1985).

[23] J. Klein, D. Perahia \& S. Warburg, "Forces between polymer-bearing surfaces undergoing shear," Nature 352 143, 1991.

[24] L.D. Landau \& E.M. Lifshitz, Theory of elasticity (Pergamon Press, Oxford, UK, 1970).

[25] L.G. Leal, "Particle motions in a viscous fluid," Annu. Rev. Fluid Mech. 12, 435-476 (1980).

[26] L.G. Leal, Laminar flow and convective transport processes: scaling principles and asymptotic analysis (Butterworth-Heineman, Newton, MA, 1992).

[27] M.J. Lighthill, "Pressure-forcing of tightly fitting pellets along fluid-filled elastic tubes," J. Fluid Mech. 34, 113-143 (1968).

[28] A.E.H. Love, A Treatise on the mathematical theory of elasticity (fourth ed. Dover 1944).

[29] A. Martin, J. Clain, A. Buguin \& F. Brochard-Wyart, "Wetting transitions at soft, sliding interfaces," Phys. Rev. E 65031605 (2002).

[30] V.C. Mow \& X.E. Guo, "Mechano-electrochemical properties of articular cartilage: their inhomogeneities and anisotropies," Annu. Rev. Biomed. Eng. 4, 175-209 (2002).

[31] V.C. Mow, M.H. Holmes \& W.M. Lai, "Fluid transport and mechanical properties of articular cartilage: a review," J. Biomechanics 17, 377-394 (1984).

[32] J. O'Donoghue, D.K. Brighton \& C.J.K. Hooke, "The effect of elastic distortions on journal bearing performance," Trans. ASME J. Lub. Tech. 89, 409-417 (1967).

[33] O. Reynolds, "On the theory of lubrication and its application to Mr. Beauchamp Tower's experiments, including an experimental determination of the viscosity of olive oil," Philos. Trans. R. Soc., London, Ser. A 177 157-234 (1886).

[34] T.W. Secomb, R. Hsu \& A.R. Pries, "A model for red blood cell motion in glycocalyx-lined capillaries," Am. J. Physiol. 274, H1016-1022 (1998).

[35] T.W. Secomb, R. Skalak, N. Özkaya \& J.F. Gross, "Flow of axisymmetric red blood cells in 
narrow capillaries," J. Fluid Mech. 163, 405-423 (1986).

[36] K. Sekimoto \& L. Leibler, "A mechanism for shear thickening of polymer-bearing surfaces: elasto-hydrodynamic coupling," Europhys. Lett. 23 113-117 (1993).

[37] A.P.S. Selvadurai (Ed.), Mechanics of Poroelastic Media, Solid Mechanics and its Application series, vol.35, Wolters Kluwer Academic Publishers (1996).

[38] J.M. Skotheim \& L. Mahadevan, "Dynamics of poroelastic filaments," Proc. R. Soc. London Ser. A 460, 1995-2020 (2004a).

[39] J.M. Skotheim \& L. Mahadevan, "Soft lubrication," Phys. Rev. Lett. 92, 245509 (2004b).

[40] H.A. Stone et al., in preparation.

[41] F. Takemura, S. Takagi, J. Magnaudet \& Y. Matsumoto, Drag and lift forces on a bubble rising near a vertical wall in a viscous fluid," J. Fluid Mech. 461, 277-300 (2002).

[42] R.I. Tanner, "An alternative mechanism for the lubrication of synovial joints," Phys. Med. Biol. 11 119-127 (1966).

[43] H. Tözeren \& R. Skalak, "The steady flow of closely fitting incompressible elastic spheres in a tube," J. Fluid Mech. 87, 1-16 (1978).

[44] H.F. Wang, Theory of Linear Poroelasticity with Applications to Geomechanics and Hydrogeology (Princeton University Press, 2000).

[45] W. Wang \& K.H. Parker, "The effect of deformable porous surface layers on the motion of a sphere in a narrow cylindrical tube," J. Fluid. Mech. 283, 287-305 (1995).

[46] S. Weinbaum, X. Zhang, Y. Han, H. Vink \& S. Cowin, "Mechanotransduction and flow across the endothelial glycocalyx," PNAS 100, 7988-7995 (2003).

[47] Wolfram Research, Inc., Mathematica, Version 5.0, Champaign, IL (2003). 
Figures 


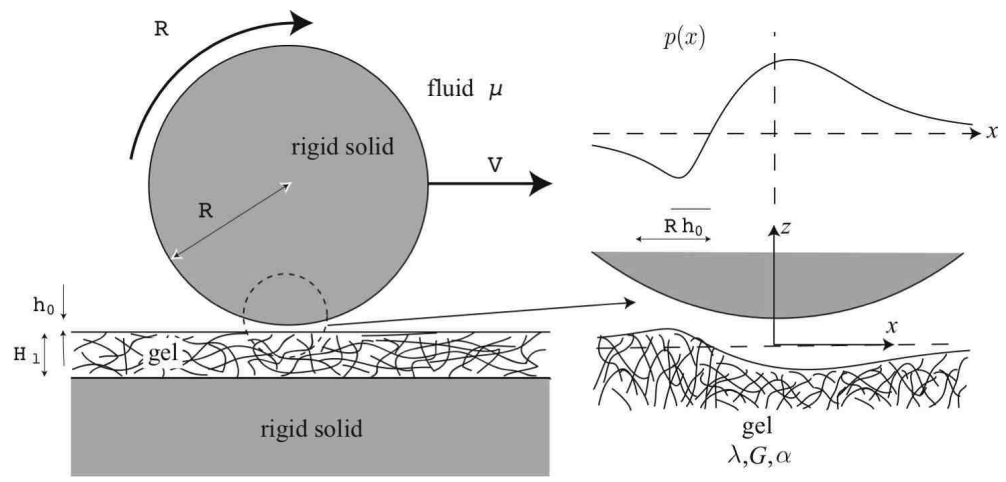

FIG. 1: A solid cylinder moves through a liquid of viscosity $\mu$ above a thin gel layer of thickness $H_{l}$ that covers a rigid solid substrate. The asymmetric pressure distribution pushes down on the gel when the fluid pressure in the gap is positive while pulling up the gel when the pressure is negative. The asymmetric traction breaks the symmetry of the gap thickness profile, $h(x)$, thus giving rise to a repulsive force of hydrodynamic origin. The pressure profile and gap thickness shown here are calculated for a thin elastic layer (乌III) for a dimensionless deflection $\eta=10$. 


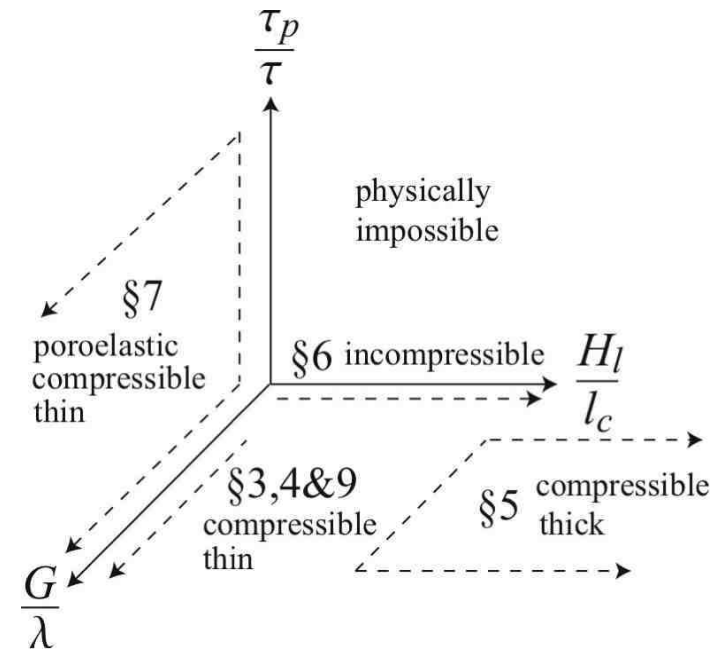

FIG. 2: Overview of the different geometries and elastic materials considered. $G$ and $\lambda$ are the Lamé coefficients of the linear elastic material, where $G / \lambda=0$ corresponds to an incompressible material, $H_{l}$ is the depth of the elastic layer coating a rigid surface, $l_{c}$ is the contact length, $\tau_{p}$ is the time scale over which stress relaxes in a poroelastic medium (a material composed of an elastic solid skeleton and an interstitial viscous fluid), and $\tau=l_{c} / V$ is the time scale of the motion. \III treats normal-tangential coupling of non-conforming contacts coated with a thin compressible elastic layer. $₫ \mathbb{I V}$ treats normal-tangential coupling of higher order degenerate contacts coated with a thin compressible elastic layer. $\$$ treats normal-tangential coupling of non-conforming contacts coated with a thick compressible elastic layer. $\$ \mathrm{VI}$ treats normal-tangential coupling of non-conforming contacts coated with an incompressible elastic layer. \$VII treats normal-tangential coupling of non-conforming contacts coated with a thin compressible poroelastic layer. normal-tangential coupling of non-conforming contacts between a rigid solid and a cylindrical shell. $\S \mathrm{IX}$ treats elastohydrodynamic effects due to coating a journal bearing with a thin compressible elastic layer. $\llbracket \mathbb{X}$ treats elastohydrodynamic effects for 3-dimensional flows using scaling analysis. 


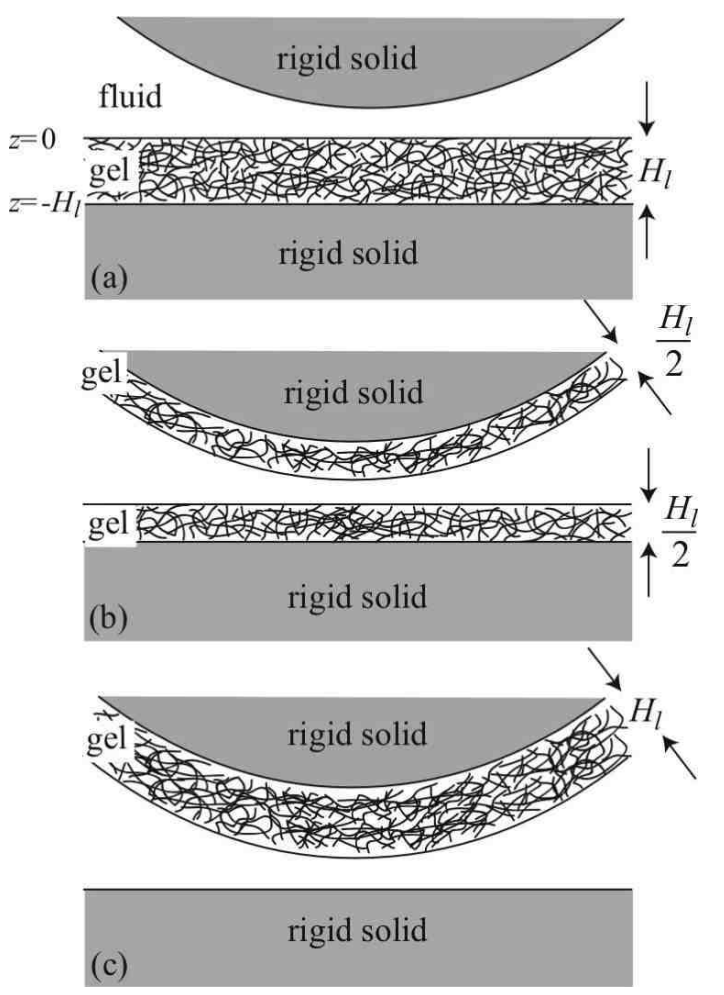

FIG. 3: Schematic diagrams of mathematically identical configurations. Configuration (a) is treated in the text. 

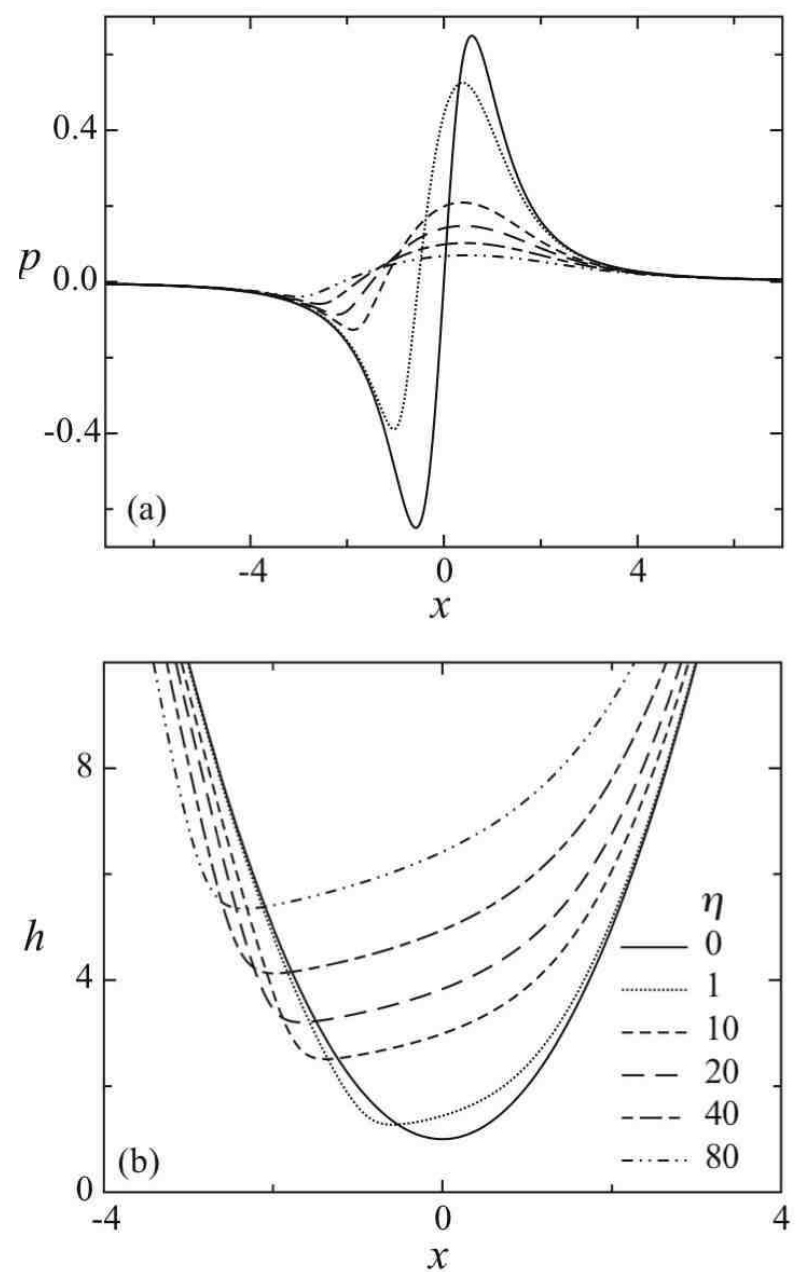

FIG. 4: (a) Pressure $p(x)$ as a function of $\eta$. (b) Gap thickness profile, $h(x)=1+x^{2}+\eta p$ as a function of $\eta$. The initially parabolic gap thickness profile is broken and the maximum value of the pressure decreases as $\eta$ increases. 

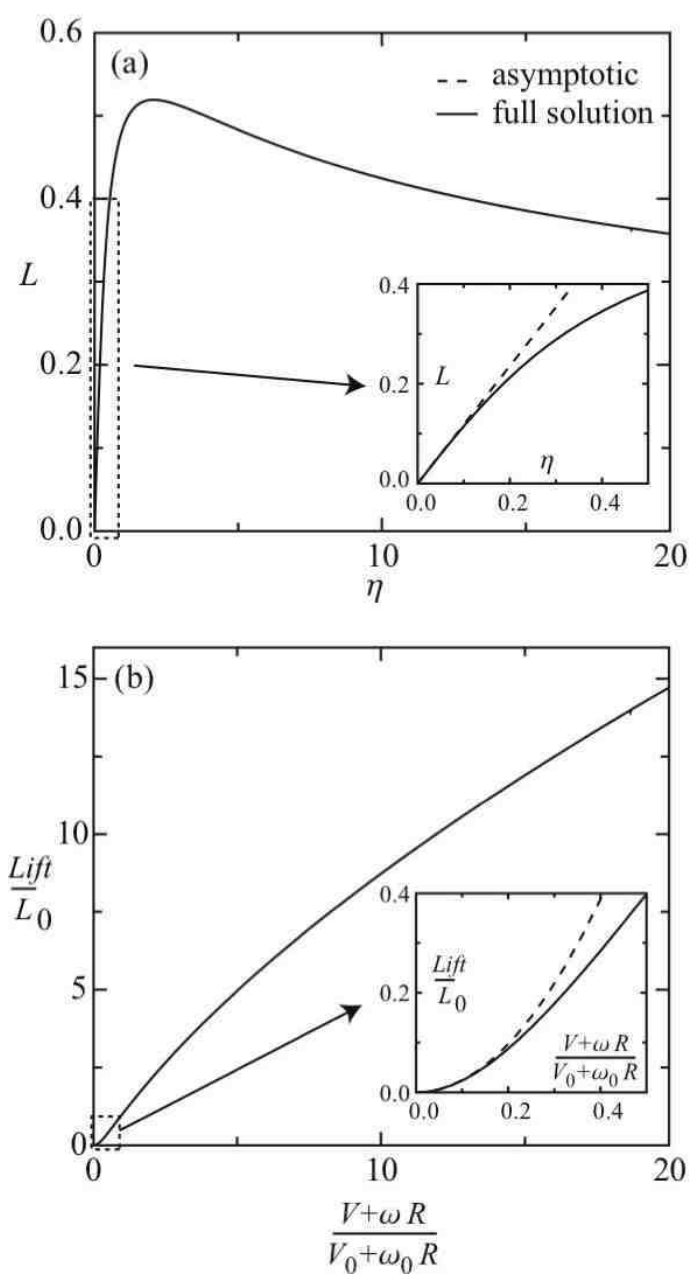

FIG. 5: (a) Dimensionless lift per unit length, $L$, plotted against $\eta$, the softness parameter. $L$ has a maximum at $\eta=2.06$ which is the result of a competition between symmetry breaking (dominant for $\eta \ll 1$ ) and decreasing pressure (dominant for $\eta \gg 1$ ) due to increasing the gap thickness. For small $\eta$ asymptotic analysis yields $L=\frac{3 \pi}{8} \eta$, which matches the numerical solution. (b) The dimensional lift force, Lift, is quadratic in the velocity for small velocities while being roughly linear for large velocities. $V_{0}+\omega_{0} R=\frac{h_{0}^{5 / 2}(2 G+\lambda)}{\sqrt{2 R} H_{l} \mu}$ is the velocity and rate of rotation at which $\eta=1$, and $L_{0}$ is corresponding lift. 

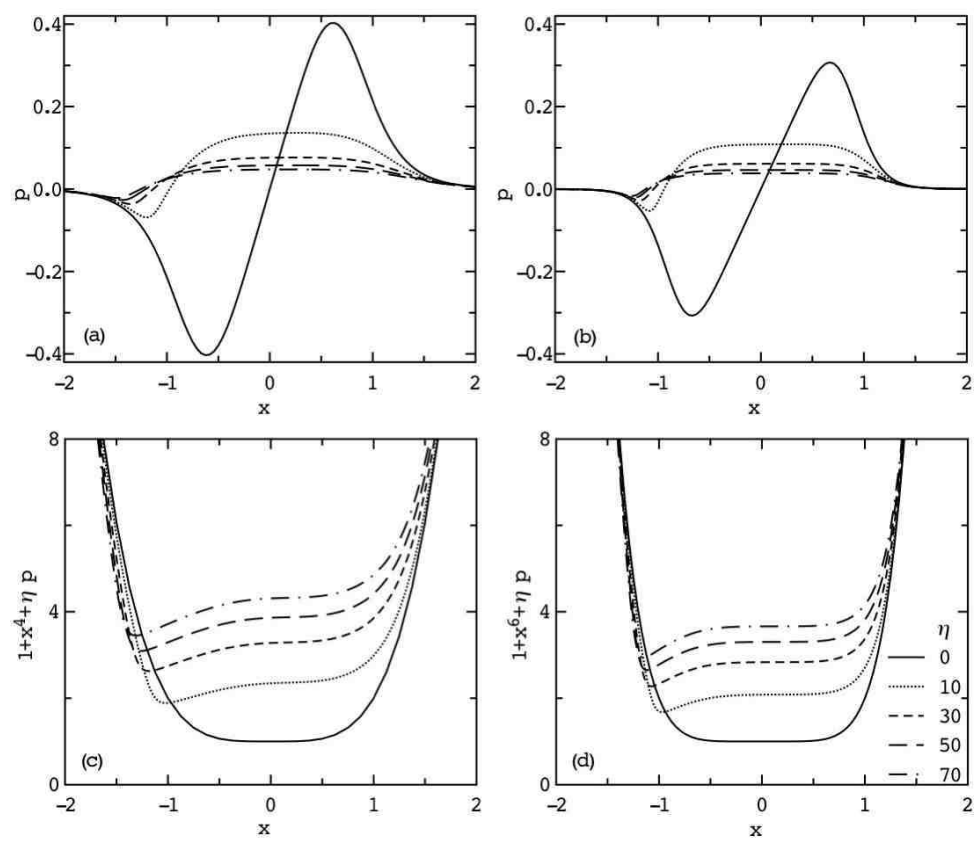

FIG. 6: Pressure distribution and gap thickness profile for degenerate contacts corresponding to a gap thickness profile of $h=1+x^{2 n}+\eta p$ with $n=2$ (a), (c), and $n=3$ (b), (d). 


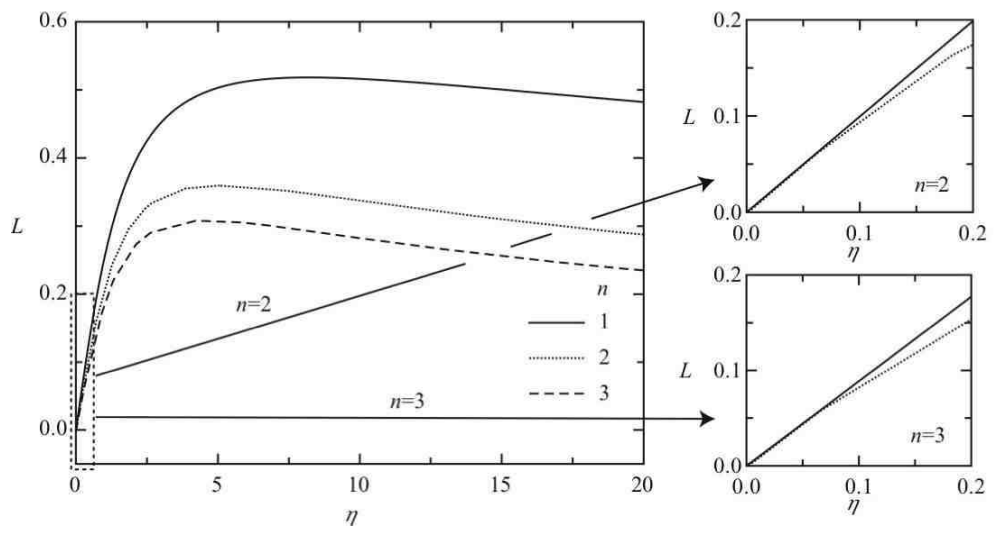

FIG. 7: Dimensionless lift for $h=1+x^{2 n}+\eta p$ where $n=1,2,3$. The curves are similar, however, they can not be rescaled to a universal curve. 


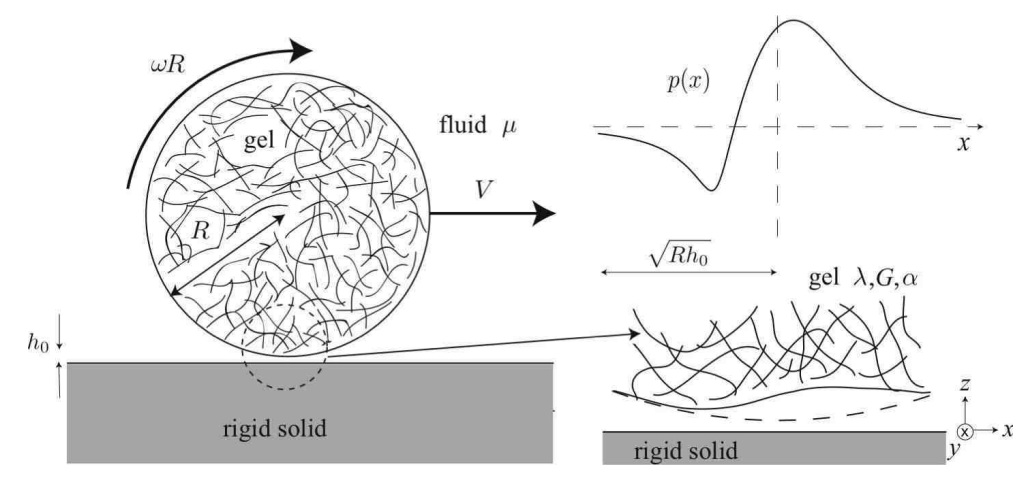

FIG. 8: Schematic diagram of a gel cylinder moving through a liquid over a rigid solid substrate. The asymmetric pressure distribution pushes on the gel when the fluid pressure in the gap is positive while pulling on the gel when the pressure is negative. This breaks the symmetry of the gap thickness profile, $h(x)$, and gives rise to a repulsive force of elastohydrodynamic origin. The dashed line in the lower right hand denotes the undeformed location of the gel cylinder. 

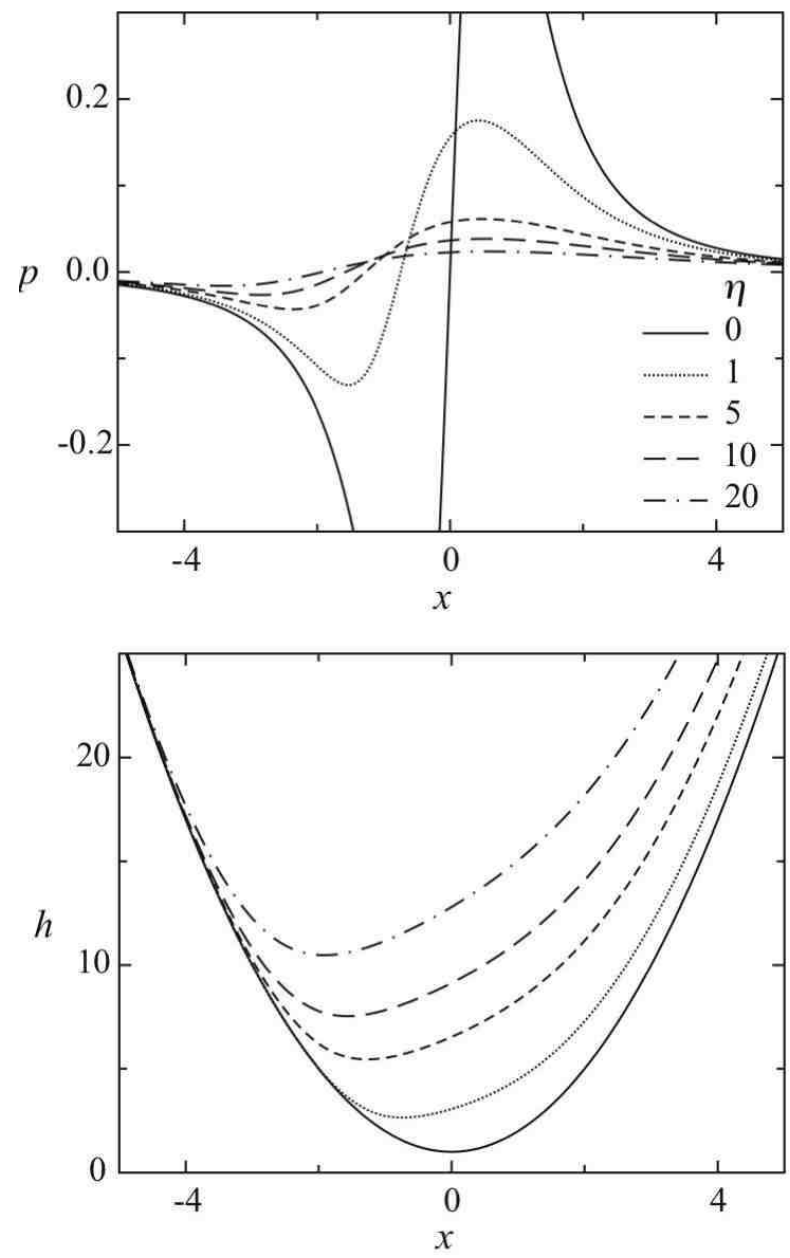

FIG. 9: Gap thickness $h$ and pressure $p$ as a function of $\eta$ for a soft cylindrical gel slider. We note that while the pressure distribution is localized to the region near the point of closest contact, the change in gap thickness is spread out due to the logarithmic nature of the Green's function of a line contact: $h=1+x^{2}+\eta \int d x^{\prime} p\left(x^{\prime}\right) \log \left[\frac{Y}{\left(x-x^{\prime}\right)^{2}}\right]$. 


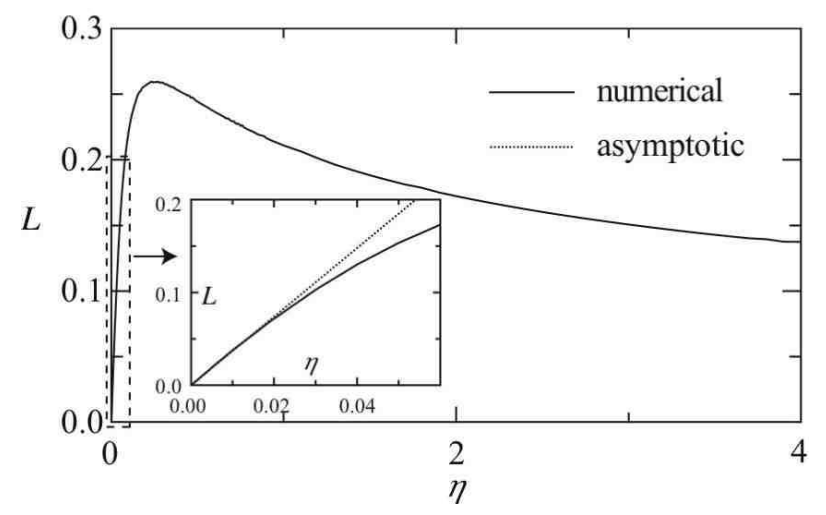

FIG. 10: Dimensionless lift as a function of $\eta$ a measure of the increase in gap thickness for a soft cylindrical gel slider. For $\eta \ll 1, L=\frac{3 \pi^{2}}{8} \eta$. 

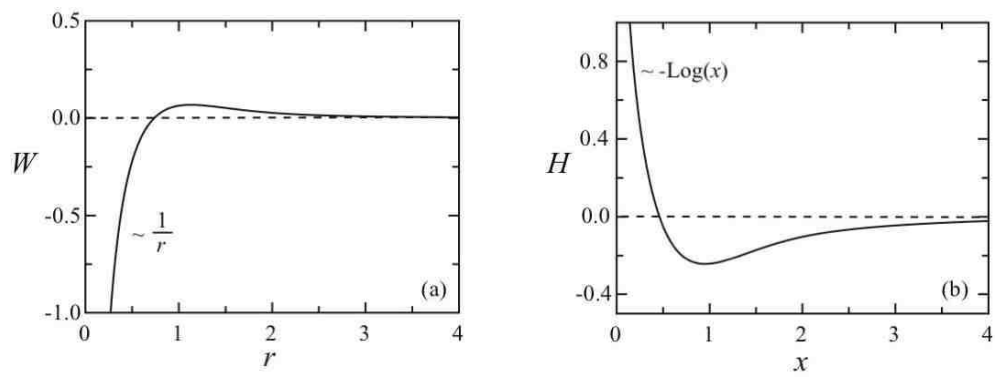

FIG. 11: Green's function for a point force (a) and a line load (b) acting on an incompressible layer of dimensionless thickness $\zeta=\frac{H_{l}}{l_{c}}=1$. 

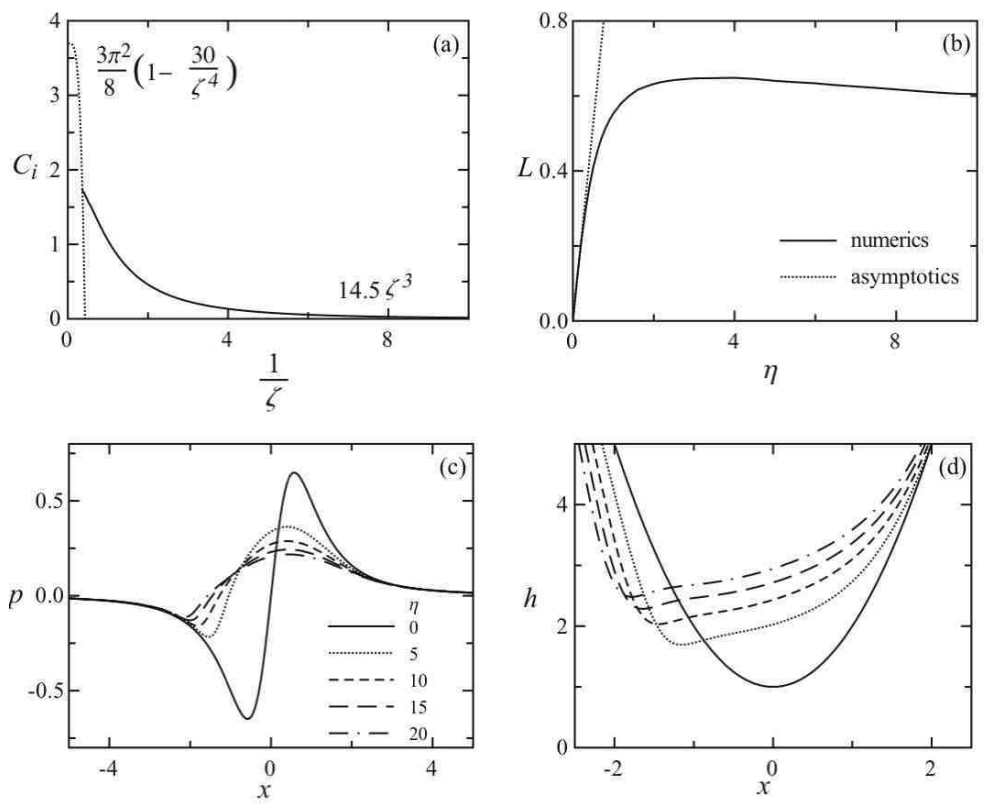

FIG. 12: For $\eta \ll 1, L=C_{i}(\zeta) \eta$ where $C_{i}(\zeta)$ is shown in (a). (b) shows the dimensionless lift per unit length, $L$, as a function of $\eta$ for $\zeta=\sqrt{h_{0} R} / H_{l}=1$. (c) and (d) show pressure, $p$, and gap thickness, $h$, as a function of $\eta$. As the thickness of the layer decreases the presence of the undeformed substrate below is increasingly felt and the layer stiffens. In the linear regime a stiffer layer results in a smaller deformation and concomitant decrease in lift. 

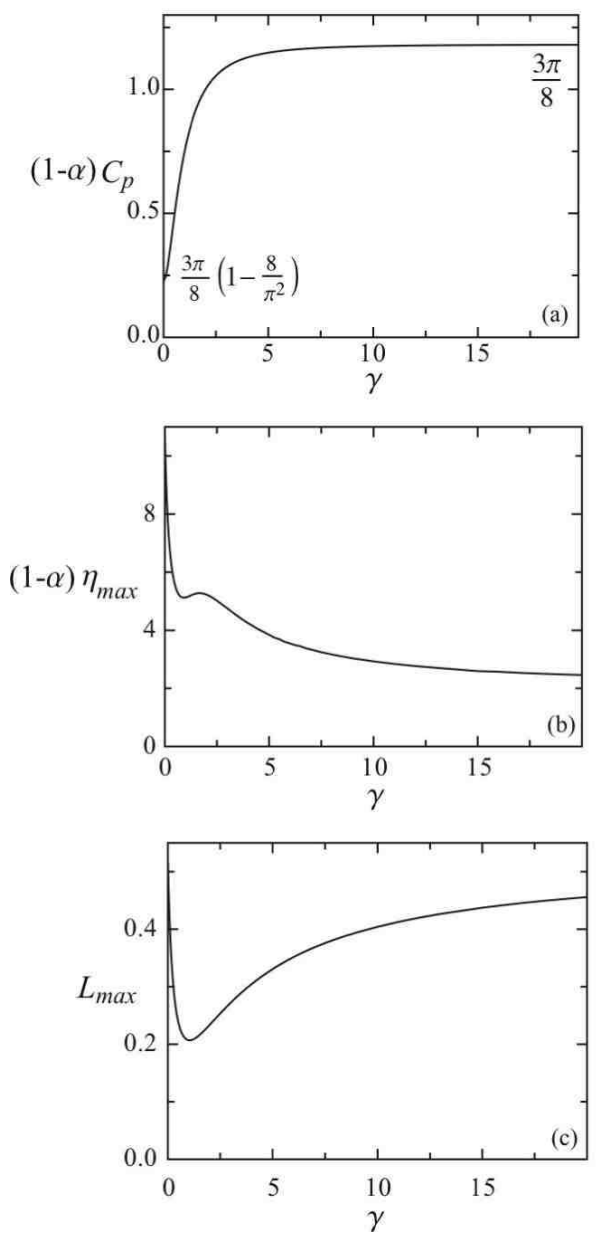

FIG. 13: For $\eta \ll 1, L=C_{p}(\gamma) \eta$ where $\gamma$ is the ratio of translational to poroelastic time scales, and $C_{p}(\gamma)$ is shown in (a). (b) $\eta_{\max }$, the value of $\eta$ at which the lift is maximum, plotted against $\gamma$. (c) The maximum lift $L_{\max }$ as a function of $\gamma$. After scaling $L$ and $\eta, \frac{L\left[\frac{\eta}{\eta_{\max }(\gamma)}\right]}{L_{\max }(\gamma)}$, the curves can be almost perfectly collapsed onto a single curve. 


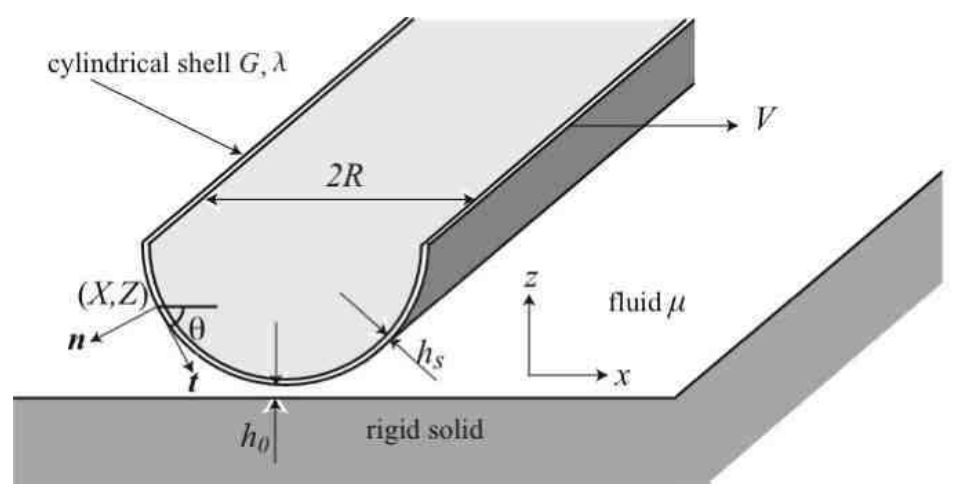

FIG. 14: Schematic diagram of a half-cylinder of radius $R$, thickness $h_{s}$ and Lamé coefficients $\mu$ and $\lambda$ moving at a velocity $V$ while completely immersed in a fluid of viscosity $\mu$. The edges of the half-cylinder are clamped at a distance $R+h_{0}$ from the surface of an undeformed solid. $\theta$ denotes the angle between the tangent to the surface and the $x$-axis. $(X(s), Z(s))$ are the laboratory frame coordinates of the half-cylinder as a function of the arc-length coordinate $s$. 


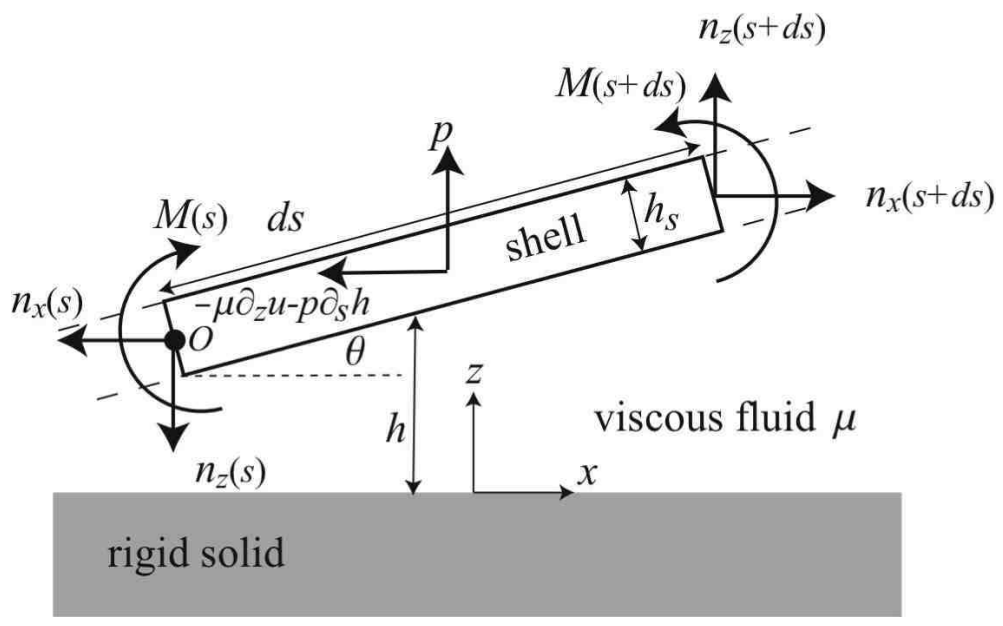

FIG. 15: Schematic of the torque and force balance for a bent cylindrical shell of thickness $h_{s}$ and Lamé coefficients $G$ and $\lambda$ subject to a traction $\left(-\mu \partial_{z} u-p \partial_{x} h, p\right)$ applied by a viscous fluid. $x$ and $z$ are coordinates in the reference frame of the rigid solid, while $s$ is the arc-length coordinate in the shell. $M(s)=\frac{G(\lambda+G) h_{s}^{3}}{3(\lambda+2 G)} \partial_{s s} \theta$ is the bending moment. 

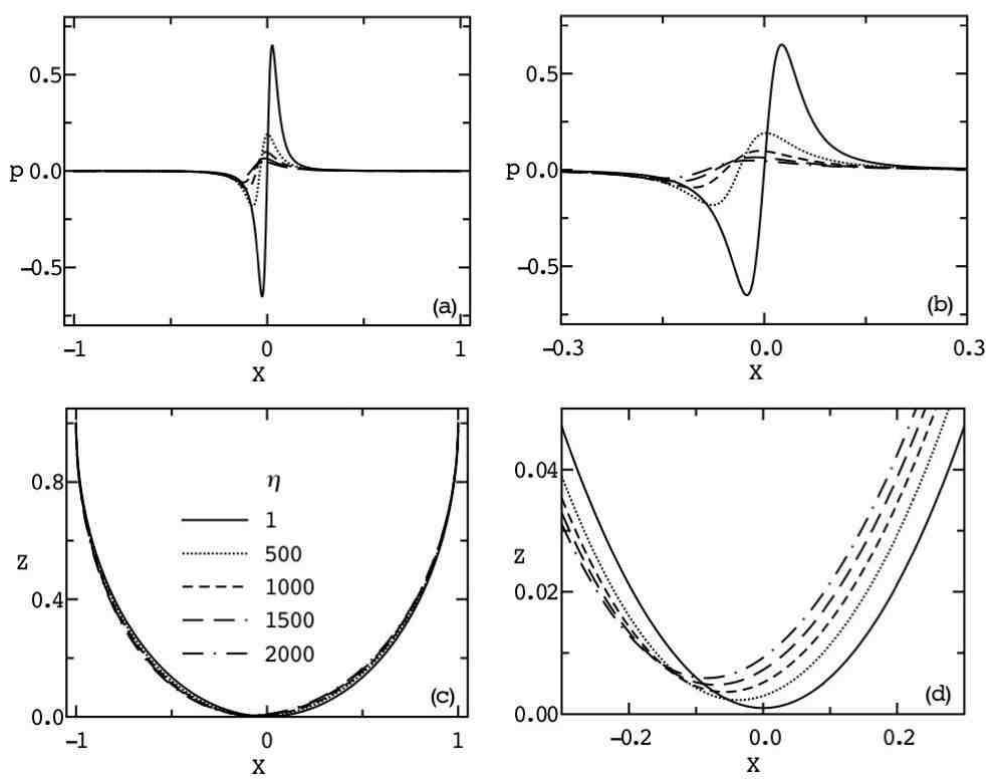

FIG. 16: (a),(b) pressure distribution, $p(X)$, as a function of the softness, $\eta$. As $\eta$ increases the asymmetry of the pressure distribution increases and the maximum pressure decreases. (c),(d) shape of the sheet, where $X(s)$ and $Z(s)$ are the coordinates of the center line in the laboratory frame. We see that the point of nearest contact is pulled back and the symmetry of the profile is broken by the forces exerted by the fluid on the cylindrical shell. $\frac{h_{0}}{R}=10^{-3}$. 

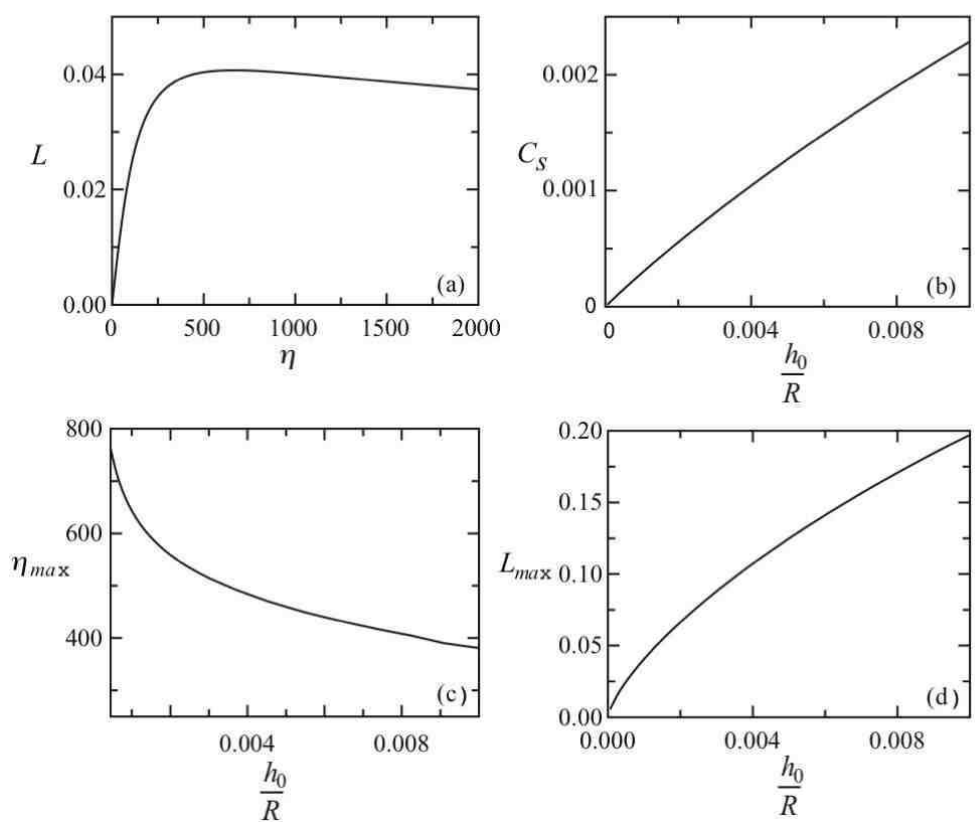

FIG. 17: (a) dimensionless lift for a cylindrical shell for $\frac{h_{0}}{R}=10^{-3}$. For $\eta<100$. $L=C_{s}\left(\frac{h_{0}}{R}\right) \eta$, where $C_{s}$ is shown in (b). The form of $L\left(\eta, \frac{h_{0}}{R}\right)$ can be almost perfectly collapsed onto a single curve after appropriately scaling the $\eta, L$ axes, i.e. $\frac{L\left[\frac{\eta}{\eta_{\max }\left(h_{0} / R\right)}\right]}{L_{\max }\left(h_{0} / R\right)}$, where $\eta_{\max }$ and $L_{\max }$ are shown in (c) and (d) respectively. 


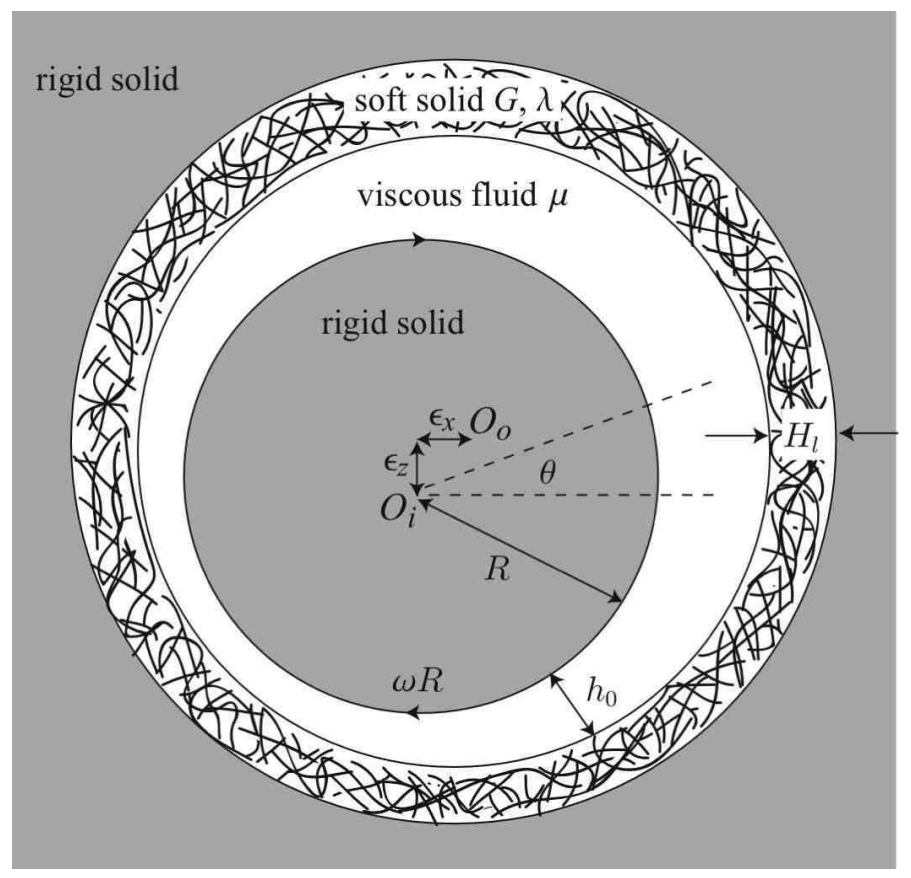

FIG. 18: Schematic diagram of the modified journal bearing geometry in which the larger cylinder of radius $R+H_{l}+h_{0}$ has been coated by a soft solid of thickness $H_{l}$ having Lamé coefficients $G$ and $\lambda$. The larger cylinder's axis is located a distance $\epsilon_{x}$ in the $x$-direction and a distance $\epsilon_{z}$ in the $z$-direction from the axis of the inner cylinder of radius $R$. The average gap thickness is $h_{0}$. 


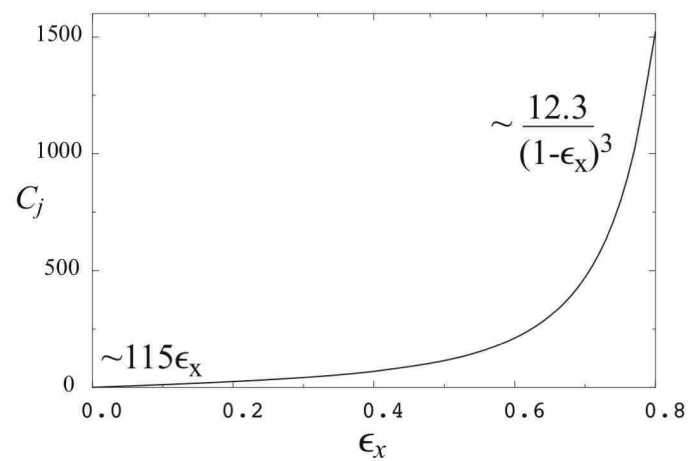

FIG. 19: For small $\eta$ the dimensionless horizontal force, $L=C_{j}\left(\epsilon_{x}\right) \eta$, where the coefficient $C_{j}$ is plotted above. 

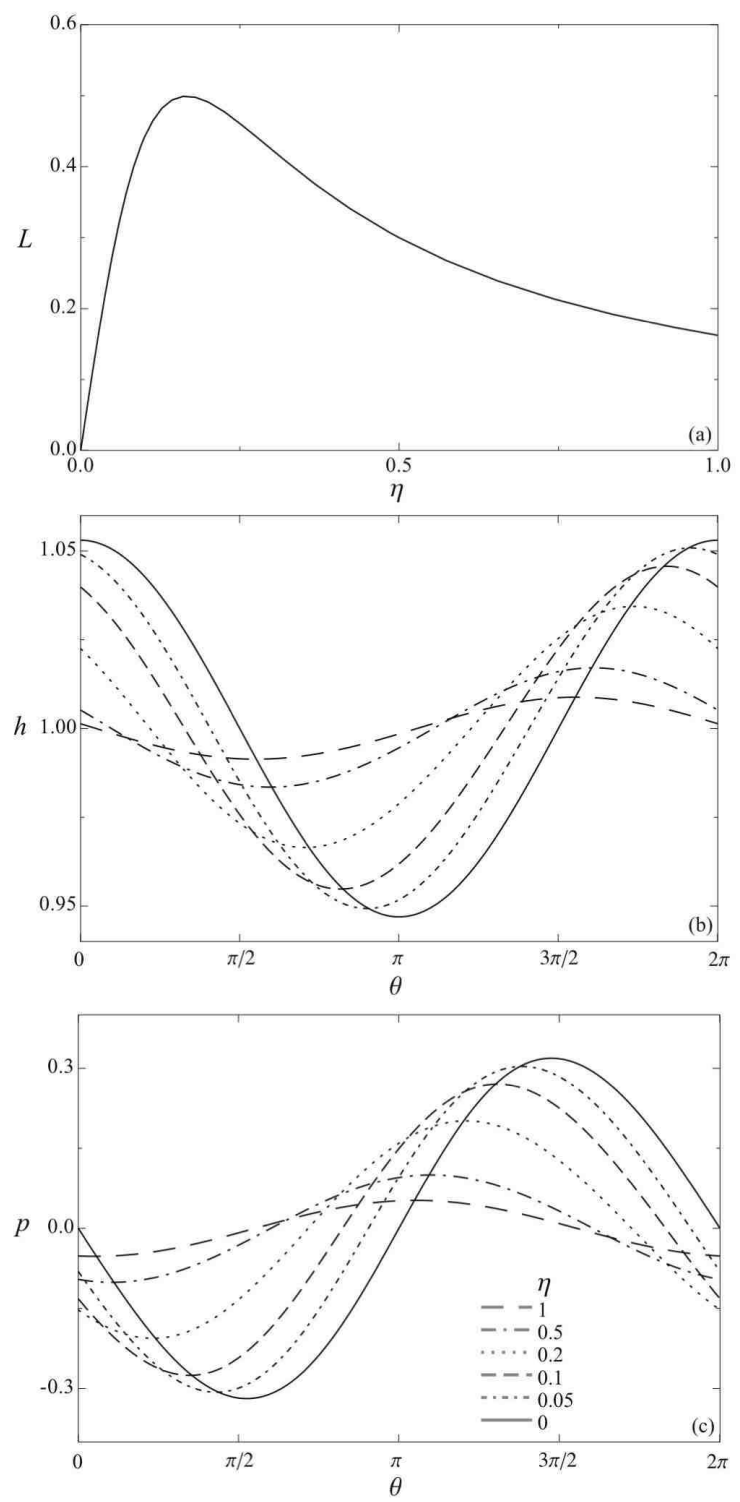

FIG. 20: (a) Dimensionless horizontal force, $L$, acting on the inner cylinder as a function of $\eta$, a measure of the surface deflection; (b) the corresponding gap thickness profiles; (c) the corresponding pressure profiles. $\epsilon_{z}=0, \epsilon_{x}=0.053$. 
Tables 
TABLE I: Summary of results for small surface deflections. ${ }^{*}$ Upper row corresponds to $n=2$, while the lower row corresponds to $n=3$ and the undeformed dimensionless gap thickness profile is $h=1+x^{2 n}$.

\begin{tabular}{|c|c|c|c|}
\hline Geometry & Material & Surface displacement & Lift force/unit length \\
\hline Thin layer & $\begin{array}{l}\text { Compressible } \\
\text { elastic solid }\end{array}$ & $\sqrt{2} \frac{\mu V}{2 G+\lambda} \frac{H_{l} R^{1 / 2}}{h_{0}^{3 / 2}}$ & $\frac{3 \sqrt{2} \pi}{4} \frac{\mu^{2} V^{2}}{2 G+\lambda} \frac{H_{l} R^{3 / 2}}{h_{0}^{7 / 2}}$ \\
\hline $\begin{array}{l}\text { Thin layer with } \\
\text { degenerate contact* }\end{array}$ & $\begin{array}{l}\text { Compressible } \\
\text { elastic solid }\end{array}$ & $\begin{array}{l}\frac{\mu V}{2 G+\lambda} \frac{H_{l} R^{3 / 4}}{h_{0}^{7 / 4}} \\
\frac{\mu V}{2 G+\lambda} \frac{H_{l} R^{5 / 6}}{h_{0}^{11 / 6}}\end{array}$ & $\begin{array}{c}\frac{351 \pi}{784 \sqrt{2}} \frac{\mu^{2} V^{2}}{2 G+\lambda} \frac{H_{l} R^{9 / 4}}{h_{0}^{17 / 4}} \\
0.8859 \frac{\mu^{2} V^{2}}{2 G+\lambda} \frac{H_{l} R^{5 / 2}}{h_{0}^{9 / 2}}\end{array}$ \\
\hline Soft slider & Elastic solid & $\frac{1}{2 \pi} \frac{\mu V(\lambda+2 G)}{G(\lambda+G)} \frac{R}{h_{0}}$ & $\frac{3 \pi^{2}}{8} \frac{\mu^{2} V^{2}(\lambda+2 G)}{G(\lambda+G)} \frac{R^{2}}{h_{0}^{3}}$ \\
\hline Thickness $\sim \sqrt{R h_{0}}$ & $\begin{array}{l}\text { Incompressible } \\
\text { elastic solid }\end{array}$ & $\frac{1}{2 \pi} \frac{\mu V}{G} \frac{R}{h_{0}}$ & $\frac{C_{i}(\zeta)}{2 \pi} \frac{\mu^{2} V^{2}}{G} \frac{R^{2}}{h_{0}^{3}}$ \\
\hline Thin layer & Poroelastic & $\sqrt{2}(1-\alpha) \frac{\mu V}{2 G+\lambda} \frac{H_{l} R^{1 / 2}}{h_{0}^{3 / 2}}$ & $C_{p}(\gamma)(1-\alpha) \frac{\mu^{2} V^{2}}{2 G+\lambda} \frac{H_{l} R^{3 / 2}}{h_{0}^{7 / 2}}$ \\
\hline Cylindrical shell & Elastic solid & $3 \sqrt{2} \pi^{2} \frac{\mu V(\lambda+2 G)}{G(\lambda+G)} \frac{R^{7 / 2}}{h_{s}^{3} h_{0}^{1 / 2}}$ & $6 \sqrt{2} \pi^{2} C_{s}\left(\frac{h_{0}}{R}\right) \frac{\mu^{2} V^{2}(\lambda+2 G)}{G(\lambda+G)} \frac{R^{9 / 2}}{h_{s}^{3} h_{0}^{5 / 2}}$ \\
\hline $\begin{array}{l}\text { Journal bearing } \\
\text { thin layer }\end{array}$ & Elastic solid & $\frac{H_{l} \mu R^{2} \omega}{h_{0}^{2}(2 G+\lambda)}$ & $C_{j}\left(\epsilon_{x}\right) \frac{\mu \omega^{2} R^{2}}{2 G+\lambda} \frac{H_{l} R^{3}}{h_{0}^{5}}$ \\
\hline
\end{tabular}


TABLE II: Summary of results for small surface deflections and spherical sliders.

\begin{tabular}{|l|l|c|}
\hline Geometry & Material & Lift force \\
\hline \hline Thin layer & Compressible & $\frac{\mu^{2} V^{2} H_{l} R^{2}}{h_{0}^{3}(2 G+\lambda)}$ \\
\hline Thin layer with & elastic solid & \\
\hline degenerate contact & elastic solid & \\
\hline Soft slider & Elastic solid & $\frac{\mu^{2} V^{2}}{G} \frac{R^{5 / 2}}{h_{0}^{5 / 2}}$ \\
\hline Thickness $\gtrsim \sqrt{R h_{0}}$ & Incompressible & $\frac{\mu^{2} V^{2}}{G} \frac{R^{5 / 2}}{h_{0}^{5 / 2}}$ \\
\hline Thickness $\ll \sqrt{R h_{0}}$ & Incompressible & $\frac{\mu^{2} V^{2}}{G} \frac{H_{l} R^{3 / 2}}{h_{0}^{5 / 2}}$ \\
\hline Thin layer & elastic solid & \\
\hline Cylindrical Shell & Elastic solid & $\frac{\mu^{2} V^{2}}{G} \frac{R^{4}}{h_{s}^{5 / 2} h_{0}^{3 / 2}}$ \\
\hline$h_{s} \gg h_{0}$ & & \\
\hline Cylindrical Shell & Elastic solid & $\frac{\mu^{2} V^{2}}{G} \frac{R^{5 / 2}}{h_{s} h_{0}^{3 / 2}}$ \\
$h_{s} \lesssim h_{0}$ & & \\
\hline Journal bearing & elastic solid & $\frac{\mu \omega^{2} R^{2}}{2 G+\lambda} \frac{H_{l} R^{4}}{h_{0}^{5}}$ \\
\hline
\end{tabular}

